\title{
Natural and vaccine-induced antibody and cellular responses against emerging SARS-CoV-2 variants of concern
}

Donal T. Skelly

University of Oxford

Adam C. Harding

University of Oxford

Javier Gilbert-Jaramillo

University of Oxford

Michael L. Knight

University of Oxford

Stephanie Longet

University of Oxford

Anthony Brown

University of Oxford

Sandra Adele

University of Oxford

Emily Adland

University of Oxford

Helen Brown

University of Oxford

Medawar Laboratory Team

Medawar Laboratory Team

Tom Tipton

University of Oxford

Lizzie Stafford

University of Oxford

Síle A. Johnson

University of Oxford

Ali Amini

University of Oxford

OPTIC Clinical Group

OPTIC Clinical Group

Tiong Kit Tan 
University of Oxford

\section{Lisa Schimanski}

University of Oxford

Kuan-Ying A. Huang

University of Oxford

Pramila Rijal

University of Oxford

PITCH Study Group

PITCH Study Group

University of Oxford

University of Oxford

John Frater

University of Oxford

Philip Goulder

University of Oxford

Chris Conlon

University of Oxford

Katie Jeffery

University of Oxford

Christina Dold

University of Oxford

Andrew J. Pollard

University of Oxford

Alain R. Townsend

University of Oxford

Paul Klenerman

University of Oxford

Susanna J. Dunachie

University of Oxford

Eleanor Barnes

University of Oxford

Miles W. Carroll

University of Oxford

William James ( $\square$ william.james@path.ox.ac.uk)

University of Oxford https://orcid.org/0000-0002-2506-1198

\section{Biological Sciences - Article}

Keywords: vaccines, SARS-CoV-2, immunization 
Posted Date: February 12th, 2021

DOl: https://doi.org/10.21203/rs.3.rs-224655/v1

License: (9) This work is licensed under a Creative Commons Attribution 4.0 International License. Read Full License

Version of Record: A version of this preprint was published at Nature Communications on August 17th, 2021. See the published version at https://doi.org/10.1038/s41467-021-25167-5. 


\section{Vaccine-induced immunity provides more robust heterotypic immunity than natural infection to emerging SARS-CoV-2 variants of concern.}

Donal T. Skelly1,4,5*, Adam C. Harding2*, Javier Gilbert-Jaramillo2, Michael L. Knight2, Stephanie Longet3,11, Anthony Brown1, Sandra Adele1, Emily Adland17, Helen Brown1, Medawar Laboratory Team, Tom Tipton3,11, Lizzie Stafford16, Síle A. Johnson5,12, Ali Amini1,5,9, OPTIC Clinical Group, Tiong Kit Tan13, Lisa Schimanski13,14, Kuan-Ying A. Huang15, Pramila Rijal13,14, PITCH Study Group, CMORE/PHOSP-C Group, John Frater1,5, Philip Goulder17, Chris Conlon16, Katie Jeffery5, Christina Dold6,8 Andrew J. Pollard6,8, Alain R. Townsend13,14, Paul Klenerman 1,5,8,9, Susanna J. Dunachie1,5,7,10 Eleanor Barnes 1,5,8,9, Miles W. Carroll3,11\#, William S. James2\#+

1. Peter Medawar Building for Pathogen Research, Nuffield Department of Medicine, University of Oxford, UK

2. Sir William Dunn School of Pathology, University of Oxford, Oxford, UK

3. Public Health England, Porton Down, UK

4. Nuffield Department of Clinical Neurosciences, University of Oxford, Oxford, UK

5. Oxford University Hospitals NHS Foundation Trust, Oxford, UK

6. Oxford Vaccine Group, Department of Paediatrics, University of Oxford, Oxford, UK

7. Mahidol-Oxford Tropical Medicine Research Unit, Bangkok, Thailand

8. NIHR Oxford Biomedical Research Centre, Oxford, UK

9. Translational Gastroenterology Unit, Nuffield Department of Medicine, University of Oxford, Oxford, UK

10. Centre for Tropical Medicine and Global Health, Nuffield Department of Medicine, University of Oxford, Oxford, UK

11. Wellcome Centre for Human Genetics, University of Oxford, Oxford, UK

12. Medical Sciences Division, University of Oxford, Oxford, UK

13. MRC Human Immunology Unit, MRC Weatherall Institute of Molecular Medicine, University of Oxford, UK

14. Centre for Translational Immunology, Chinese Academy of Medical Sciences, Oxford Institute, University of Oxford, Oxford, UK

15. Division of Pediatric Infectious Diseases, Department of Pediatrics, Chang Gung Memorial Hospital and College of Medicine, Chang Gung University, Taoyuan, Taiwan

16. Nuffield Department of Medicine, University of Oxford, Oxford, UK 
17. Peter Medawar Building for Pathogen Research, Department of Paediatrics, University of Oxford, Oxford, UK

*These authors contributed equally

\# These authors contributed equally

+ Corresponding author 


\section{Summary}

Both natural infection with SARS-CoV-2 and immunization with a number of vaccines induce protective immunity. However, the ability of such immune responses to recognize and therefore protect against emerging variants is a matter of increasing importance. Such variants of concern (VOC) include isolates of lineage B1.1.7, first identified in the UK, and B1.351, first identified in South Africa. Our data confirm that VOC, particularly those with substitutions at residues 484 and 417 escape neutralization by antibodies directed to the ACE2-binding Class 1 and the adjacent Class 2 epitopes but are susceptible to neutralization by the generally less potent antibodies directed to Class 3 and 4 epitopes on the flanks RBD. To address this potential threat, we sampled a SARS-CoV-2 uninfected UK cohort recently vaccinated with BNT162b2 (Pfizer-BioNTech, two doses delivered 18-28 days apart), alongside a cohort naturally infected in the first wave of the epidemic in Spring 2020. We tested antibody and T cell responses against a reference isolate (VICO01) representing the original circulating lineage B and the impact of sequence variation in these two VOCs. We identified a reduction in antibody neutralization against the VOCs which was most evident in the B1.351 variant. However, the majority of the T cell response was directed against epitopes conserved across all three strains. The reduction in antibody neutralization was less marked in post-boost vaccine-induced than in naturally-induced immune responses and could be largely explained by the potency of the homotypic antibody response. However, after a single vaccination, which induced only modestly neutralizing homotypic antibody titres, neutralization against the VOCs was completely abrogated in the majority of vaccinees. These data indicate that VOCs may evade protective neutralising responses induced by prior infection, and to a lesser extent by immunization, particularly after a single vaccine, but the impact of the VOCs on T cell responses appears less marked. The results emphasize the need to generate high potency immune responses through vaccination in order to provide protection against these and other emergent variants. We observed that two doses of vaccine also induced a significant increase in binding antibodies to spike of both SARS-CoV-1 \& MERS, in addition to the four common coronaviruses currently circulating in the UK. The impact of antigenic imprinting on the potency of humoral and cellular heterotypic protection generated by the next generation of variant-directed vaccines remains to be determined. 


\section{Acknowledgements.}

B1.351 lineage virus isolate 512Y.V2.HW.001 was kindly provided by Dr. Alex Sigal, KwaZulu-Natal Research Institute for Tuberculosis and HIV, Professor Tulio de Oliveira, KwaZulu-Natal Research Innovation and Sequencing Platform (KRISP), and the staff of the Centre for Aids Programme of Research in South Africa (CAPRISA), University of KwaZulu-Natal, Durban, South Africa. Variant B.1.1.7 was isolated and rapidly shared by Kevin Bewley and colleagues within the National Infection Service at Public Health England, Porton Down UK. The customised coronavirus ELISA plates were a gift from Meso Scale Diagnostics, Rockville, MD USA. We thank OUH COVID research nurses and ISARIC.

\section{Competing Interests:}

The authors declare no competing interests.

\section{Funding Statements:}

The views expressed in this article are those of the authors and not necessarily those of the National Health Service (NHS), the National Institutes for Health Research (NIHR), or the Medical Research Council (MRC).

This work was supported by the UK Department of Health and Social Care as part of the PITCH (Protective Immunity from T cells to Covid-19 in Health workers) Consortium, the UK Coronavirus Immunology Consortium (UK-CIC) and the Huo Family Foundation. EB and PK are NIHR Senior Investigators and PK is funded by WT109965MA and NIH (U19 I082360). SD is funded by an NIHR Global Research Professorship. M.C., S.L. and T.T. are funded by a USA FDA grants HHSF223201510104C \& 75F40120C00085 Characterization of severe coronavirus infection in humans and model systems for medical countermeasure development and evaluation. A.C.H. and W.J. are supported by University of Oxford Rapid COVID Response, for which the contribution of donors is gratefully acknowledged. DS is supported by the NIHR Academic Clinical Fellow programme in Oxford. J.G-J is supported by Ecuadorian National Government Scholarship, M.L.K. is supported by the BBSRC, A.A. is a Wellcome Clinical Research Training Fellow (216417/Z/19/Z). PK is in the National Institute for Health Research Health Protection Research Unit (NIHR HPRU) in Emerging and Zoonotic Infections (NIHR200907) at University of Liverpool in partnership with Public Health England (PHE), in collaboration with Liverpool School of Tropical Medicine and the University of Oxford. The C-MORE 
authors' work was supported by NIHR Oxford Biomedical Research Centre, British Heart Foundation (BHF) Oxford Centre of Research Excellence (RE/18/3/34214), United Kingdom Research Innovation. This project is also funded by the Medical Research Council and Department of Health and Social Care/ National Institute for Health Research Grant (MR/V027859/1) ISRCTN number 10980107, as part of the collaborative research programme entitled PHOSP-COVID Post-hospitalisation COVID-19 study: a national consortium to understand and improve long-term health outcomes. 


\section{Introduction}

The emergence of new lineages of SARS-CoV-2 on three continents towards the end of 2020 , and their rapid expansion at the expense of the previously dominant lineages, poses significant challenges to public health (WHO SARS-CoV-2 Variants) $)^{1}$. In order to address these challenges effectively, there is an urgent need to understand the biological consequences of the mutations found in these lineages, and the consequential impact on their susceptibility to current control measures, including vaccines, drugs and nonpharmaceutical interventions.

All three variants share the N501Y substitution in the receptor-binding domain (RBD) of spike glycoprotein $(S)$, which increases binding affinity of $S$ with the virus's cellular receptor, ACE2 ${ }^{2}$ (see Figure 1). Lineage B.1.1.7, first identified in the UK in September $2020\left({ }^{1}\right)$, is characterized by additional mutations in S, such as deletion of residues $69 \& 70$ and the P681H substitution for which plausible effects on the virus biology are proposed, as well as five other mutations in S, a premature stop codon in ORF8, three substitutions and a deletion in ORF1 and two amino acid substitutions in nucleoprotein (N) of as-yet unknown significance. Lineage B.1.351 ${ }^{3}$ was first identified in November 2020 in South Africa and is characterized by two additional substitutions of likely significance in RBD, namely, K417N and E484K. The former is predicted to disrupt a salt bridge with D30 of ACE2, a characteristic of SARS-CoV-2 in distinction to SARS-CoV-1, but may not impact on binding, whereas the latter, which might disrupt the interaction of RBD with K31 of hACE2, may enhance ACE2 binding ${ }^{24}$. Very recently (Jan 2021) E484K has been detected first in lineage B1.1.7 in the UK ${ }^{5}$ and subsequently in lineages A23.1, B.1 and B.1.177, as well as in imported cases of B.1.51 and P. $2^{2}$. Our data confirm that VOC, particularly those such as B1.351 with substitutions at residues 484 and 417, escape neutralization by antibodies directed to the ACE2-binding Class 1 and the adjacent Class 2 epitopes, but are susceptible to neutralization by the generally less potent antibodies directed to Class 3 and 4 epitopes on the flanks of the RBD

\footnotetext{
${ }^{1}$ Preliminary genomic characterisation of an emergent SARS-CoV-2 lineage in the UK defined by a novel set of spike mutations - SARS-CoV-2 coronavirus / nCoV-2019 Genomic Epidemiology - Virological

${ }^{2}$ Updated regularly at Nextstrain / groups / neherlab / ncov / S.E484
} 
The immune correlates of protection against infection and disease caused by SARS-CoV-2 are imperfectly understood (recently reviewed by ${ }^{6}$ ). Classically, neutralization by antibody, measured by reduction in plaque or infectious foci by authentic virus in vitro is considered a major component of protection, though indirect effects of antibody, such as complement activation and opsonization may also play a role in vivo. Recent studies have demonstrated that symptomatic re-infection within six months after the first wave in the UK was very rare in the presence of anti-S or anti-N IgG antibodies 7,8 . Virus-specific lymphocytes may play an important direct role in protection, in addition to their indirect effect mediated through help to antibody-producing cells. Robust T cell immune responses (with CD4+ T cells dominating) to $\mathrm{S}, \mathrm{M}, \mathrm{N}$ and some ORF antigens are readily detected after infection, correlate with disease severity and are durable for at least several months ${ }^{9-11}$. Furthermore, CD8 depletion studies in non-human primate (NHP) challenge studies suggest T cells also play a protective role especially when antibody levels are low ${ }^{12}{ }^{13}$. Nevertheless, passive infusion of neutralizing antibody has been shown to be sufficient to mediate effective protection against SARS-CoV-2 in these rhesus macaque challenge studies ${ }^{13}$, encouraging the evaluation of both convalescent sera and monoclonal antibody (mAb) cocktails as early therapeutic interventions. In common with endemic human coronaviruses, immunity following recovery from natural infection does not appear to be always sufficient to prevent re-infection, although the increase in frequency of such cases towards the end of 2020 appears to be linked to the emergence of antigenically distinct lineages (see above).

At the time of writing (February 2021) multiple vaccines have been reported to have efficacy against COVID-19 disease in phase III clinical trials. Of these, three - BNT162b2, mRNA-1273 and Sputnik V - that were reported to have efficacies in the mid-90\% range, had also induced classical neutralising antibody titres substantially higher than those found on average in convalescent patients ${ }^{14-16}$. In contrast, one - CoronaVac - that showed approximately 50\% efficacy, had been reported to induce neutralising titres several-fold lower than those found in convalescent patients ${ }^{17}$. The two remaining vaccines, BBIP-CorV and AZD1222 (ChAdOx-1 nCoV-19), had intermediate values of both clinical efficacy and relative potency in generating neutralising antibody responses ${ }^{18,19}$. mRNA and Adenovirusvectored vaccines generate high magnitude SARS-CoV-2 multispecific CD4+ and CD8+ T cells responses. Reports of vaccines assessed in South Africa where B.1.351 dominates are 
currently emerging and include Ad26.COV2.S (single dose Ad26 vectored vaccine), Novovax (recombinant Spike/adjuvant) and CAZD1222. Each of the studies report reduced efficacy in South African populations. Vaccine correlates of protection, and the relative contribution of T cell and humoral immunity are yet to be precisely defined since detailed immune analysis in people with vaccine breakthrough infections is lacking.

In pseudotype virus assays - in which S is not chaperoned by viral membrane proteins $\mathrm{M}$ and $E$, buds at the plasma membrane rather than internally, and has an immature quaternary structure that appears to render it variably more sensitive to neutralization by antibody - it appears that convalescent sera from patients exposed to prototype strains of SARS-CoV-2, in distinction to vaccine-elicited responses, may not be effective in neutralizing lineage $B 1.351^{20,21}$. As the lineage-defining substitutions include changes in previously identified antibody epitopes and regions of $\mathrm{S}$ associated with its processing and rearrangement during cellular infection, this is a very plausible observation. In order to test whether convalescent sera and sera from vaccine recipients were similarly affected in their ability to neutralize authentic virions, we have undertaken classical neutralization assays against reference isolates of both B1.1.7 and B1.351 compared to the prototype, early pandemic, B VICO1 isolate. We find that, while cross-neutralization of B1.1.7 is only modestly reduced compared to that of the prototype strain, cross-neutralization of B1.351 is markedly reduced. This effect is particularly marked in convalescent sera and in a very minor $(<10 \%)$ subset of those that had mounted somewhat modest neutralizing responses to the vaccine.

Since viral mutations may also affect $T$ cell recognition, we evaluate the contribution of $T$ cells that target epitopes located at sites of amino acid substitution in the spike glycoproteins of VOCs. We evaluated the T cell response to peptides spanning the entire spike protein in an interferon-gamma (IFN- $\gamma$ ) ELISpot assay and found that the majority of T cell responses in recipients of two doses of the BNT162b2 vaccine are generated by epitopes that are invariant between the prototype Victoria strain and the three VOCs.

Our results suggest that reformulation of vaccines to address new variant lineages ought to be considered and indicates that seasonal re-vaccination might be required for this virus. We also show that neutralization of VOC is significantly enhanced by a second boost vaccination. There is legitimate concern that antigenic imprinting during vaccination to 
prototype $\mathrm{S}$ antigen blunts the response to variant sequences incorporated into nextgeneration vaccines. However, our results show that vaccination not only induces enhanced reactivity to $\mathrm{S}$ from endemic human coronaviruses, but also significant cross-reactivity to both SARS-CoV-1 and MERS-CoV. Encouragingly, we find evidence that the T cell epitopes that dominate responses in these same individuals, are not subject to major substitution in the three variants of concern. 


\section{Methods}

\section{Volunteer samples}

Volunteers were recruited at Oxford University Hospitals NHS Foundation Trust in ethically approved studies and included: 1) Hospitalised patients with severe COVID-19 defined as SARS-CoV-2 PCR positive and requiring in patient oxygen support, recruited under study CMORE (research ethics committee (REC): North West - Preston, REC reference 20/NW/0235); 2) Healthcare Workers (HCWs) with asymptomatic or mild symptomatic COVID-19 disease defined as SARS-CoV-2 PCR positive disease and not requiring $\mathrm{O} 2$ support/hospitalization and; 3) HCWs not known to be previously infected with SARS-CoV-2, sampled 7-17 days after receiving COVID-19 mRNA Vaccine BNT162b2, 30 micrograms, administered intramuscularly after dilution as a series of two doses (0.3 mL each) 18-28 days apart (study OPTIC: Oxford Translational Gastrointestinal Unit GI Biobank Study 16/YH/0247 [[REC at Yorkshire \& The Humber - Sheffield] 2). The study was conducted according to the principles of the Declaration of Helsinki (2008) and the International Conference on Harmonization (ICH) Good Clinical Practice (GCP) guidelines. Written informed consent was obtained for all patients enrolled in the study.

\section{Virus isolates}

Prototype isolate (PANGO lineage B) was Victoria/01/202022 (B VIC01), received at P3 from PHE Porton Down (after being supplied by the Doherty Centre Melbourne) in April 2020, passaged in VeroE6/TMPRSS2 cells, used here at P5, and confirmed identical to GenBank MT007544.1

B1.1.73 (20I/501Y.V1.HMPP1) isolate, H204820430, 2/UK/VUI/1/2020, received in Oxford at P1 from PHE Porton Down in December 2020, passaged in VeroE6/TMPRSS2 cells (NIBSC reference 100978), used here at $P 4$.

B1.351 (20I/501.V2.HV001) isolate ${ }^{23}$ was received at P3 from the Centre for the AIDS Programme of Research in South Africa (CAPRISA), Durban, in Oxford in January 2021, passaged in VeroE6/TMPRSS2 cells (NIBSC reference 100978), used here at P4.

\footnotetext{
${ }^{3}$ https://virological.org/t/preliminary-genomic-characterisation-of-an-emergent-sars-cov-2-lineage-in-the-ukdefined-by-a-novel-set-of-spike-mutations/563
} 
For all isolates, identity was confirmed by deep sequencing at the Wellcome Trust Centre for Human Genetics, University of Oxford.

\section{Microneutralization Assay (MNA)}

The study was performed in the CL3 Facility of the University of Oxford operating under license from the HSE, on the basis of an agreed Code of Practice, Risk Assessments (under ACDP) and Standard Operating Procedures. The microneutralization assay determines the concentration of antibody that produces a $50 \%$ reduction in infectious focus-forming units of authentic SARS-CoV-2 in Vero CCL81 cells, as follows. Quadruplicate serial dilutions of serum or monoclonal antibody were preincubated with a fixed dose of SARS-CoV-2 before incubation with Vero cells. A 1.5\% carboxymethyl cellulose-containing overlay was used to prevent satellite focus formation. Twenty hours post-infection, the monolayers were fixed with $4 \%$ paraformaldehyde, permeabilized with $2 \%$ Triton X-100 and stained for the nucleocapsid (N) antigen or spike (S) antigen using mAbs EY $2 A$ and EY 6A, respectively ${ }^{24}$. After development with a peroxidase-conjugated antibody and TrueBlue peroxidase substrate, infectious foci were enumerated by ELISPOT reader. Data were analysed using four-parameter logistic regression (Hill equation) in GraphPad Prism 8.3.

\section{Mesoscale Discovery (MSD) binding assays}

IgG responses to SARS-CoV-2, SARS-CoV-1, MERS and seasonal CoVs were measured using a multiplexed MSD immunoassay: the V-PLEX COVID-19 Coronavirus Panel 3 (IgG) Kit (Cat \# K15399U) from Meso Scale Diagnostics, Rockville, MD USA. A MULTI-SPOT ${ }^{\circledR}$ 96-well, 10 Spot Plate was coated with three SARS CoV-2 antigens (S, RBD, N), SARS and MERS spike trimers, as well as spike proteins from seasonal CoV OC43, HKU1, 229E, NL63 and bovine serum albumin. Antigens were spotted at $200-400 \mu \mathrm{g} / \mathrm{mL}$ (MSD ${ }^{\circledR}$ Coronavirus Plate 3). Multiplex MSD Assays were performed as per the instructions of the manufacturer. To measure IgG antibodies, 96-well plates were blocked with MSD Blocker A for 30 minutes. Following washing with washing buffer, samples diluted 1:500 in diluent buffer, as well as the reference standard and internal controls were added to the wells. After 2-hour incubation and a washing step, detection antibody (MSD SULFO-TAG ${ }^{\mathrm{TM}}$ Anti-Human IgG Antibody, $1 / 200$ ) was added. Following washing, MSD GOLD ${ }^{\text {TM }}$ Read Buffer $B$ was added and plates were read using a MESO ${ }^{\circ}$ SECTOR S 600 Reader. Statistical analysis was performed using Kruskal-Wallis one-way ANOVA. 
Victoria strain SARS-CoV2 peptides, 15-18 amino-acids overlapping by 11 amino-acids and spanning the entire spike region, were used in two pools (Mimotopes) CMV, EBV, influenza and tetanus antigens (CEFT) were used in a single pool ( $2 \mu \mathrm{g} / \mathrm{mL}$ : Proimmune, Oxford, UK). Single peptides (Mimotopes, Victoria Australia) that mapped to sites with mutations in B1.1.7 $(n=17), B 1.351(n=21)$ and P.1 $(n=22)$ with reference to Victoria strain were used in single peptides or pooled by strain.

\section{IFN- $\gamma$ T cell ELISpot assays}

Peripheral blood mononuclear cells (PBMC) were isolated by density gradient centrifugation using Lymphoprep ${ }^{T M}(p=1.077 \mathrm{~g} / \mathrm{mL}$, Stem Cell Technologies), washed twice with RPMI 1640 (Sigma, St. Louis, MO, USA) containing 10\% heat-inactivated FCS (Sigma), 1mM Pen/Strep and $2 \mathrm{mM}$ L-Glutamine (Sigma) and resuspended in R10 and counted using the Guava ${ }^{\circledR}$ ViaCount ${ }^{\mathrm{TM}}$ assay on the Muse Cell Analyzer (Luminex Cooperation). PBMCs were frozen and stored in liquid nitrogen before use.

96-well Multiscreen-I plates (Millipore, UK) were coated for 3 hours with $10 \mu \mathrm{g} / \mathrm{mL} \mathrm{GZ-4}$ anti-human IFN- $\gamma$ (Mabtech, AB, Sweden) at room temperature. PBMC were added at $2 \times 10^{5}$ cells in $50 \mu \mathrm{l}$ per well and stimulated with $50 \mu \mathrm{l}$ of SARS-CoV-2 peptide pools ( $2 \mathrm{ug} / \mathrm{mL}$ per peptide) in duplicate. R10 with DMSO (final concentration $0.4 \%$, Sigma) was used as negative control and CEFT ( $(2 \mu \mathrm{g} / \mathrm{mL}$, Proimmune)/ Concanavalin A $(5 \mu \mathrm{g} / \mathrm{mL}$ final concentration, Sigma) were used as positive control antigens. After $16-18$ hours at $37^{\circ} \mathrm{C}$ PBMC were removed and secreted IFN- $\gamma$ detected using anti-IFN- $\gamma$ biotinylated mAb at 1 $\mu \mathrm{g} / \mathrm{mL}$ (7-B6-1-biotin, Mabtech) for 2-3 hours, followed by streptavidin alkaline phosphatase at $1 \mu \mathrm{g} / \mathrm{mL}$ for $1-2$ hours (SP-3020, Vector Labs). The plates were developed using BCIP/NBT substrate (Pierce) according to the manufacturer's instructions. ELISpot plates were read using an AID ELISpot Reader (v.4.0). Results were reported as spot-forming units (SFU) $/ 10^{6}$ PBMC. Background (mean SFU in negative control wells) was subtracted from antigen stimulated wells to give the final result.

\section{Expression and purification of monoclonal antibodies}

Monoclonal antibodies used in this study were produced as previously described ${ }^{25}$. The variable heavy and light genes of these mAbs were cloned into the human IgG1, human kappa light chain or lambda light chain expression vectors (AbVec-hlgG1, AbVec-hlgKappa or 
AbVec-hlgLambda). The heavy and light antibody plasmids were transfected into the Expi293F (Gibco) cell line for expression of recombinant full-length human IgG1 according to manufacturer's protocol. Harvested supernatant was clarified at $3,000 \mathrm{~g}$ for $10 \mathrm{~min}$ and filtered $(0.22 \mu \mathrm{m})$ before affinity purification using a HiTrap MabSelect SuRe column (Cytivia, Westborough MA, USA) according to manufacturer's instruction. Eluted IgG1 were concentrated, and buffer exchanged into PBS using an Amicon Ultra-15 filter unit with 30k molecular weight cut-off. 


\section{Results}

Homotypic and Heterotypic neutralization of key SARS-CoV-2 lineages by antibody.

The primary structure of the Spike glycoprotein, and the characteristic sequence variants of the current three lineages of concern are illustrated in Figure 1. In this study, we analysed the homotypic neutralization of the prototype strain, Victoria/01/2020 (PANGO lineage A), by monoclonal antibodies (mAbs), sera from convalescent individuals following COVID-19, and recipients of the BNT162b2 (Pfizer) vaccine, which are each induced by prototypic $S$ antigen, and heterotypic neutralization by these same antibodies of two new lineages of concern (B1.1.7 and B1.351). In Figure 1, we indicate the residues of $S$ at which the respective lineage - as well as a third lineage of concern, P.1 - differ from prototype.

\section{Neutralization by sera from convalescent COVID-19 individuals}

Sera from convalescent individuals neutralized prototype virus with highly variable potency (see Figure 2a). The neutralization potency against Lentivirus (SARS-CoV-2 S) pseudotypes broadly - but imperfectly - corresponded with homotypic neutralization of authentic virus. Sera neutralized the B1.1.7 isolate with a lower potency (2-fold; 95\% CL: $1.5-3$-fold), and those with the lowest homotypic neutralizing potency had undetectable heterotypic potency. The decline in heterotypic neutralization potency against the B1.351 isolate was more marked with 5/9 having undetectably low neutralizing potency.

\section{Neutralization by sera from vaccine recipients.}

After a single dose of BNT162b2 vaccine, 10/11 sera showed homotypic neutralization potency equivalent to that of the more modest half of convalescent sera (NT50 1/100, see Figure $2 b)$. Heterotypic neutralization was undetectable in all 11 sera against B1.351.

Sera from 23/25 individuals drawn between 7 and 17 days after two doses of BNT162b2 vaccine administered 18 to 28 days apart neutralized prototype virus with high potency (NT50 >> 1/100, Figure 2c), whereas $2 / 25$ individuals showed more modest titres $(1 / 10<$ NT50 < 1/100). Sera neutralized the B1.1.7 isolate with a lower potency (2-fold; 95\% CL: 1.5 -3-fold), but in no case was neutralization undetectable. The decline in neutralization potency against the B1.351 isolate was somewhat greater on average but only the 2/25 with modest homotypic neutralization potency had undetectable heterotypic neutralizing potency. 
The data from convalescent individuals and those having received vaccine are summarized in Fig2D, showing the trend to lower neutralization potency across the three genotypes of virus, which is least marked in those receiving two doses of vaccine. The relationship of the neutralizing titre of each individual's serum to B VICO1 to their neutralizing titre against each VOC is displayed in Fig 2E and shows clear pairwise correlation. Correlation coefficients are: 0.95 (B VIC01 to B.1.1.7 HMPP1); 0.81 (B VIC01 to B1.351 HV001); and 0.80 (B1.1.7 HMPP1 to B1.351 HV001).

\section{Neutralization by monoclonal, neutralizing mAbs to the four epitopes of RBD and by reference serum}

In order to understand better the reasons for differences between the homotypic and heterotypic neutralization potency of polyclonal human sera seen above, we made use of a panel of six, epitope-mapped neutralizing monoclonal antibodies (NmAbs, Figure 2F, and Fig S1) ${ }^{25-28}$. We have devised a "squirrel" diagram to help visualise the binding sites of the various mAbs on the RBD (Figure $2 \mathrm{~d}$ ). One NmAb, FI 3A, a Class 1 RBD mAb (binds to the left side of the head of the squirrel) whose homotypic NT50 is of the order of $1 \mathrm{nM}$, is largely unaffected by the changes in B1.1.7 HMPP1 (NT50 = 1.365) but does not neutralize B1.351 HV001. Two other NmAbs, GR 12C and C121 that are Class 2 RBD binding mAb (that bind to the right side of the head of the squirrel), and which have homotypic NT50 $0.1 \mathrm{nM}$, show some reduced effectiveness in neutralizing B1.1.7 HMPP1 and have lost all potency against B1.351 HW001. This might be expected, as class 2 antibodies bind to an epitope that includes residue 484 (reviewed by Barnes et al and ${ }^{29}$ ). In contrast, NmAb FD 11A and S309, which are Class 3 RBD mAb, that bind to the right haunch of the squirrel, and EY 6A, Class 4 $\mathrm{mAb}$, that binds to the left haunch of the squirrel, appears to be unaffected by the mutations in the VOCs.

Polyclonal responses generated by different individuals to natural infection or in response to vaccination may include a varying proportion of antibodies to these and other neutralization epitopes. We also noted significant deviations in heterotypic neutralization potency against a currently approved reference serum 20/130 (NIBSC, Fig 2G). While homotypic NT50 was $1 / 918.2$ (95\% CL 1/729.6 - 1/1,165), close to the result of 1:1280 quoted on the 20/130 data sheet, neutralization of B1.1.7 HMPP1 was enhanced by approximately 10 -fold, but of B1.351 HV01 was reduced by $>10-$ fold. 


\section{Binding of antibodies to Alpha and Beta Coronavirus proteins}

We probed the antibody-binding properties of sera from vaccinated, convalescent and control sera using a customised MSD coronavirus antigen array ELISA (Figure 3). We observed that sera from individuals receiving two doses of the Pfizer vaccine showed significantly higher binding to both SARS-CoV-2 spike and RBD compared to those receiving single dose and to convalescent individuals one month after infection (Fig $3 \mathrm{~A}$, and B, respectively). The absence of antibody binding to $\mathrm{N}$ (Fig $3 \mathrm{C}$ ) confirms that all vaccinated individuals are naïve for SARS-CoV-2 infection.

There was significant antibody binding to both SARS-CoV-1 and MERS spike protein in vaccinated and COVID-19 convalescent individuals compared to the negative control sera (Fig 3D \& E, respectively). This was particularly marked for SARS-CoV-1 reactivity in fully vaccinated individuals, suggesting that vaccine can induce a broad response to widely shared epitopes, such as those exemplified by EY 6A (see above) and CR3022 $2^{30}$.

We also screened for antibody binding to the spike antigen of the four common coronaviruses circulating in the UK (Fig $3 \mathrm{~F}-\mathrm{I})$. There is a significant increase in binding to all four, particularly to the Betacoronavirus clade A isolates, HKU1 and OC43, in vaccinated and COVID-19 convalescent sera $(p<0.0001)$. Binding to the Alphacoronavirus isolates, $229 \mathrm{E}$ and, to a lesser extent, NL63S was also greater in the post-boost vaccinees, but not in convalescent sera.

\section{T cell responses to Spike antigens in prototype strain Victoria and VOCs}

Following two doses of BNT162b2, spike-specific T cells were detected in all individuals (mean magnitude 561, range 110-1717 SFC/106 PBMC) against spike antigens covering the Victoria strain, assessed in IFN- $\gamma$ ELISpot assays (Fig 4A). Assessing the contribution of T cells that target epitopes located at the site of B1.1.7, B1.351 and P.1 specific mutation sites we find that T cells targeted epitopes spanning all spike mutation sites (Fig 4B) (8, 9 and 10 epitopes in lineage B1.1.7, B1.351 and P.1 respectively). In each individual, T cells targeted $0-19$, mean 6) epitopes located at mutation sites. The overall contribution of these, to the total spike specific response (mean magnitude and range) is $13 \%(0-67 \%), 14 \%(0-44 \%)$ and 10\% (0-29\%) respectively (Fig 4C). 


\section{Prediction of heterotypic neutralization by immunoassay}

Authentic virus neutralization assays require specialist staff and facilities that are not widely available, and access to reference isolates of virus that are laborious to distribute. Accordingly, we asked whether high throughput ELISA-style immunoassays could provide a degree of predictive value for heterotypic neutralization. Using the Mesoscale discovery (MSD) assay, we investigated the relationship between the binding activity of serum to homotypic SARS-CoV-2 S and its heterotypic neutralization potency against both SARS-CoV-2 VOCs, and its ability to bind to other pandemic and endemic human coronaviruses. The correlation coefficients are summarized and the individual data are shown in Figure 5. Unsurprisingly, binding to SARS-CoV-2 S correlates very strongly with binding to SARS-CoV-2 S in convalescent sera, though surprisingly less well in post-boost vaccinees, who, reassuringly, showed no reactivity to SARS-CoV-2 N. S binding in vaccinees predicted homotypic and heterotypic neutralization moderately $\left(0.5>\right.$ Spearman $\left.r>0.4,{ }^{*}\right)$. $S$ binding offered no predictive value for either homotypic or heterotypic neutralization in convalescent sera. Interestingly, binding activity in post-vaccine sera to SARS-CoV-2 R predicted binding to MERS-CoV S very well $\left(r=0.69,{ }^{* * *}\right)$ but not to SARS-CoV-1 S. While SARS-CoV-2 S binding activity in vaccinee sera predicted binding to the $\mathrm{S}$ of one endemic beta coronavirus (OC43 S r $=0.57,{ }^{* *}$; HKU1 S, r $=0.44,{ }^{*}$ ) it did not predict binding to the other two endemic coronaviruses.

Conversely, RBD binding predicted neutralization of VIC01 very well (Spearman $r=0.57,{ }^{* *}$ ), but neutralization of B1.1.7 less well $\left(r=0.48,{ }^{*}\right)$ and of B1.351 not at all $(r=0.35, n s)$, and showed an inverted relationship in binding to other epidemic coronavirus S proteins (SARSCoV-1 S, r = 0.67, ***; MERS-CoV S, $r=0.52, *$; data not shown). 


\section{Discussion}

Our results show that overall, both binding and neutralization by antibodies induced by prototypic S protein is diminished to S from recent variants of concern (VOC); B1.351 to a greater extent than B1.1.7. This broad trend masks both qualitative and quantitative differences in antibody responses by individuals, whose serum may contain differing proportions of antibodies to neutralizing epitopes that we show here are sometimes conserved between strains and always reduced in VOCs, particularly to B1.351.

Although, in principle, neutralization results using pseudotype viruses and in vitro binding assays may not reliably predict the potency of heterotypic immune protection afforded by natural infection or immunization by prototypic SARS-CoV-2, our results using authentic virus largely substantiate provisional conclusions recently made using such data.

Encouragingly, we find that the majority of T cell responses in recipients of two doses of the BNT162b2 vaccine are generated by epitopes that are invariant between the prototype and two of the current variants of concern (B1.1.7 and B1.351). T cell responses to SARS-CoV-2 are known to target a wide range of regions in spike ${ }^{31}, 8$. Moreover, in over $90 \%$ of these recipients, heterotypic neutralizing titres (NT50) remain comfortably above the level associated with immune protection in recent vaccine trials. However, in a majority of individuals whose homotypic neutralization titres were more modest - including over $50 \%$ of convalescent COVID-19 individuals and recipients of a single dose of vaccine - heterotypic neutralization dropped to negligible levels. This loss of cross-neutralization was particularly notable against B1.351 with potential implications for vaccine efficacy in populations where this VOC dominates and when only moderate levels of $S$ antibodies are generated after vaccination.

It should be noted that neutralization escape, observed in a well of a micro-titre plate, is not direct evidence of vaccine failure ${ }^{32-34}$. Non-neutralising antigen-specific antibodies, $T$ cells and innate lymphocytes clearly have the potential to contribute to vaccine efficacy ${ }^{35}$. The acceptance that prior infection with influenza virus results in reduced disease against subsequent infection with heterosubtypic strains, in both human and animal challenge studies, provides further evidence that cellular components and non-neutralising antibodies make an important contribution to protection ${ }^{33}$. We also note that the recent South African and UK Novavax vaccine clinical trials showed $50-80 \%$ protective efficacy against 
infection for the B1.351 and B1.1.7 VOC respectively. Furthermore, cases of vaccinated individuals requiring hospitalization due to severe disease were extremely rare for these VOCs. Ongoing analysis of real-world vaccine roll out will illuminate the extent of vaccine breakthrough with VOCs.

Nevertheless, our results re-emphasize the urgent need to deploy the most effective vaccine strategies as widely and rapidly as possible in order to provide population protection against the emerging lineages of concern of SARS-CoV-2. Our findings show clearly that the weaker responses generated for example by natural infection or single doses of vaccine, do not provide adequate cross-neutralization. The question remains, however, whether antigenic imprinting to prior infection or vaccination with prototypic antigen will pose a significant obstacle to the newly formulated vaccine candidates, incorporating sequences derived from the variants of concern. 


\section{Figure Legends}

Figure 1. Sequence variation in Spike glycoprotein. The open reading frame encoding Spike is illustrated, with the position of key features of processing and function indicated to approximate scale (residue number indicated above). During cotranslational translocation to the ER, the short leader peptide (LP) is proteolytically removed. Following folding, trimer assembly and glycosylation in the ER and Golgi, the trans-Golgi localized protease, furin, cleaves the boundary between the S1 and S2 polypeptides. Following binding of the receptor-binding domain (RBD, cyan) to ACE2 on host cells, cell-surface TMPRSS2 proteolytically cleaves the S2' site, facilitating conformational changes to spike that result in fusion of the virus envelope with the plasma membrane. Variant residue positions are indicated below, and their approximate location on the $\mathrm{S}$ polypeptide is indicated. Residue identities are shown at each of these positions for a prototype isolate, Victoria/01/2020 (VIC01, PANGO lineage B), and at each position in the three lineages of interest (B1.1.7, B1.351, and P.1) at which the respective lineage differs from prototype. $\Delta$ indicates deletion of one or more residues. Note, there are lineage-defining substitutions outside RBD, in the $\mathrm{N}$-terminal domain (NTD) and C-terminal domain (CTD) of S1 (dark blue), and in S2 (tan). These may include changes that directly or indirectly affect antibody-mediated neutralization by loss or altered dynamics of epitope, respectively.

Figure 2. Homotypic and heterotypic neutralization of key SARS-CoV-2 lineages by antibody. The potency of neutralization was determined by a focus-forming unit microneutralization assay against authentic virus of prototype $B$ (Victoria) lineage and isolates of lineages B1.1.7 and B1.351 (see methods). Detailed neutralization curves and confidence limits on the estimates of NT50 are given in the supplementary data. A. Neutralization by convalescent sera stratified by high medium and low antibody responses, as determined by Lentivirus (SARS-CoV-2 S) pseudotype assay against prototype, B1.1.7 and B1.351 isolates. B. Neutralization by sera from recipients of a single dose of BNT162b2 vaccine, and $\mathbf{C}$. Neutralization by sera from recipients of both prime and boost doses of BNT162b2 vaccine. D. Homotypic and heterotypic neutralization potencies of the three sources of antibody against the three isolates, shown by individual and sub-population mean and SD of NT50 values. E. the relationship of the potency of heterotypic to homotypic neutralization for each of 25 post-boot vaccine recipient sera F. Neutralization by a panel of 
monoclonal antibodies binding to four distinct epitopes of RBD. (upper left) A space-filling model of prototype RBD (PDB 6YZ5) created in PyMOL, shows the residue of the mutations present in the B.1.1.7 and B.1.351 lineages in blue. (upper middle and upper right; same aspect and reverse aspect as the space-filling model, respectively) A cartoon of a "squirrel" we devised to illustrate the RBD. Markings on the squirrel cartoon show the epitopes of the RBD antibodies used in this study (classes 1 to $4^{26}$ ).

Figure 3. Binding assays. IgG antibodies specific to; A-C SARS-CoV-2 (S, RBD, N), D-E SARSCoV-1 S, MERS S, F-I 229E S, HKU1 S, NL63 S, OC43S, were measured using an MSD technology platform customised array. Sera analysed was from vaccinees (post-prime and post-boost), COVID-19 convalescent sera (one-month post PCR+) and a cohort of negative sera collected between 2014 and 2018. Green, blue and brown dots show samples which displayed a high, medium or low neutralising ability, respectively and included in MNA. Statistical difference between the groups was performed using a Kruskal-Wallis one-way ANOVA. Vaccinees postprime $n=11$; vaccinees post-boost $n=25$; negatives $n=103$; COVID-19 convalescents $n=86$.

Figure 4: ELISpot responses to prototype, B1.1.7, B1.351 and P1. T cell responses were measured using IFN- $\gamma$ ELISpot assays in 24 healthy volunteers, 7-17 days after receiving the 2nd dose of BNT162b2. A. T cell responses to 15-18-mer peptides overlapping by 11 aminoacids and spanning the entire spike region, alongside responses to summed peptides from the Victoria strain that mapped to sites with mutations in B1.1.7 ( $\mathrm{n}=17$ peptides), B1.351 ( $n=21$ peptides) and P.1 ( $n=22$ peptides). B. Percentage contribution of SARS-CoV-2 variants $B 1.1 .7, B 1.351$ and $P 1$ to the total T cell spike response in each of the 24 volunteers. Variants shown as a percentage of total spike response to Victoria strain as determined by ex vivo IFN- $\gamma$ ELISpot C. T cell responses to 22 individual peptides in Victoria strain that have corresponding to mutations in B1.1.7, B1.351 and P1 variants. Each bar represents one volunteer with a positive response (defined as a response to the peptide minus the background that was greater than a twice the background). $\mathrm{N}=24, \mathrm{SFC} / 10^{6} \mathrm{PBMC}=\mathrm{spot}$ forming cells per million peripheral blood mononuclear cells, with background subtracted.

Figure 5. Cross-correlation of immune parameters. Pairwise Spearman correlation analyses were undertaken between the value of binding of post-boost vaccinee or convalescent serum antibody to SARS-CoV-2 S, as determined by the MSD immunoasssay platform (see Figure 3), and the homotypic and heterotypic neutralizing titre of the same sera (see Figure 
2) and the MSD-determined binding to SARS-CoV-2 RBD and N, and to the S protein of other coronaviruses. Spearman's $r$ parameter, in the associated two-tailed $\mathrm{P}$ value and its interpretation are given for each pairwise comparison. In the panels, below, the MSD values (left axis) and NT50 values (right axis) for each individual are plotted against the corresponding MSD value for SARS-CoV-2 S. 
Supplementary data

Figure S1.

individual neutralization curves for each antibody/isolate combination 


\section{References}

1. Rambaut, A. et al. Preliminary genomic characterisation of an emergent SARS-CoV-2 lineage in the UK defined by a novel set of spike mutations. virological.org (2020).

2. Starr, T. N. et al. Deep Mutational Scanning of SARS-CoV-2 Receptor Binding Domain Reveals Constraints on Folding and ACE2 Binding. Cell 182, 1295-1310.e20 (2020).

3. Tegally, H. et al. Emergence and rapid spread of a new severe acute respiratory syndrome-related coronavirus 2 (SARS-CoV-2) lineage with multiple spike mutations in South Africa. medRxiv (2020).

4. Verkhivker, G. M., Agajanian, S., Oztas, D. \& Gupta, G. Computational Analysis of Protein Stability and Allosteric Interaction Networks in Distinct Conformational Forms of the SARS-CoV-2 Spike D614G Mutant: Reconciling Functional Mechanisms through Allosteric Model of Spike Regulation. bioRxiv 2021.01.26.428331 (2021). doi:10.1101/2021.01.26.428331

5. Collier, A. et al. SARS-CoV-2 B.1.1.7 escape from mRNA vaccine-elicited neutralizing antibodies 12 Dami. (2021). doi:10.21203/RS.3.RS-156101/V1

6. Dai, L. \& Gao, G. F. Viral targets for vaccines against COVID-19. Nature Reviews Immunology 21, 73-82 (2020).

7. Mahase, E. Covid-19: Past infection provides $83 \%$ protection for five months but may not stop transmission, study finds. BMJ 372, n124 (2021).

8. Lumley, S. F. et al. Antibody Status and Incidence of SARS-CoV-2 Infection in Health Care Workers. N. Engl. J. Med. (2020). doi:10.1056/nejmoa2034545

9. Ogbe, A. et al. T cell assays differentiate clinical and subclinical SARS-CoV-2 infections from cross-reactive antiviral responses. medRxiv 2020.09.28.20202929 (2020). doi:10.1101/2020.09.28.20202929

10. Dan, J. M. et al. Immunological memory to SARS-CoV-2 assessed for up to 8 months after infection. Science (80-. ). 371, eabf4063 (2021).

11. Peng, Y. et al. Broad and strong memory CD4+ and CD8+ T cells induced by SARS-CoV2 in UK convalescent individuals following COVID-19. Nat. Immunol. 21, 1336-1345 (2020).

12. $\mathrm{Yu}$, J. et al. DNA vaccine protection against SARS-CoV-2 in rhesus macaques. Science (80-. ). 369, 806 LP-- 811 (2020).

13. McMahan, K. et al. Correlates of protection against SARS-CoV-2 in rhesus macaques. Nature 1-5 (2020). doi:10.1038/s41586-020-03041-6

14. Logunov, D. Y. et al. Safety and efficacy of an rAd26 and rAd5 vector-based heterologous prime-boost COVID-19 vaccine: an interim analysis of a randomised controlled phase 3 trial in Russia. Lancet 0, (2021).

15. Baden, L. R. et al. Efficacy and Safety of the mRNA-1273 SARS-CoV-2 Vaccine. N. Engl. J. Med. (2020). doi:10.1056/nejmoa2035389 
16. Polack, F. P. et al. Safety and Efficacy of the BNT162b2 mRNA Covid-19 Vaccine. N. Engl. J. Med. 383, 2603-2615 (2020).

17. Zhang, Y. J. et al. Immunogenicity and safety of a SARS-CoV-2 inactivated vaccine in healthy adults aged 18-59 years: Report of the randomized, double-blind, and placebo-controlled phase 2 clinical trial. medRxiv 2020.07.31.20161216 (2020). doi:10.1101/2020.07.31.20161216

18. Barrett, J. R. et al. Phase $1 / 2$ trial of SARS-CoV-2 vaccine ChAdOx1 nCoV-19 with a booster dose induces multifunctional antibody responses. Nat. Med. (2020). doi:10.1038/s41591-020-01179-4

19. Folegatti, P. M. et al. Safety and immunogenicity of the ChAdOx1 nCoV-19 vaccine against SARS-CoV-2: a preliminary report of a phase $1 / 2$, single-blind, randomised controlled trial. Lancet 1-13 (2020). doi:10.1016/S0140-6736(20)31604-4

20. Wibmer, C. K. et al. SARS-CoV-2 501Y.V2 escapes neutralization by South African COVID-19 donor plasma. bioRxiv 2021.01.18.427166 (2021). doi:10.1101/2021.01.18.427166

21. Muik, A. et al. Neutralization of SARS-CoV-2 lineage B.1.1.7 pseudovirus by BNT162b2 vaccine-elicited human sera. bioRxiv 2021.01.18.426984 (2021).

doi:10.1101/2021.01.18.426984

22. Caly, L. et al. Isolation and rapid sharing of the 2019 novel coronavirus (SARS-CoV-2) from the first patient diagnosed with COVID-19 in Australia. Med. J. Aust. (2020). doi:10.5694/mja2.50569

23. Cele, S. et al. Escape of SARS-CoV-2 501Y.V2 variants from neutralization by convalescent plasma. medRxiv 2021.01.26.21250224 (2021). doi:10.1101/2021.01.26.21250224

24. Huang, K.-Y. A. et al. Breadth and function of antibody response to acute SARS-CoV-2 infection in humans. bioRxiv 2020.08.28.267526 (2020). doi:10.1101/2020.08.28.267526

25. Huang, K.-Y. A. et al. Breadth and function of antibody response to acute SARS-CoV-2 infection in humans. bioRxiv 2020.08.28.267526 (2020). doi:10.1101/2020.08.28.267526

26. Barnes, C. O. et al. Structures of Human Antibodies Bound to SARS-CoV-2 Spike Reveal Common Epitopes and Recurrent Features of Antibodies. Cell 182, 828842.e16 (2020).

27. Robbiani, D. F. et al. Convergent antibody responses to SARS-CoV-2 in convalescent individuals. Nature 584, 437-442 (2020).

28. Pinto, D. et al. Cross-neutralization of SARS-CoV-2 by a human monoclonal SARS-CoV antibody. Nature 583, 290-295 (2020).

29. Wang, Z. et al. mRNA vaccine-elicited antibodies to SARS-CoV-2 and circulating variants 2 3. bioRxiv 2021.01.15.426911 (2021). doi:10.1101/2021.01.15.426911 
30. Ter Meulen, J. et al. Human monoclonal antibody combination against SARS coronavirus: synergy and coverage of escape mutants. PLoS Med. 3, e237 (2006).

31. Grifoni, A. et al. Targets of T Cell Responses to SARS-CoV-2 Coronavirus in Humans with COVID-19 Disease and Unexposed Individuals. Cell 181, 1489-1501.e15 (2020).

32. Gooch, K. E. et al. Heterosubtypic cross-protection correlates with cross-reactive interferon-gamma-secreting lymphocytes in the ferret model of influenza. Sci. Rep. 9, 1-10 (2019).

33. Sridhar, S. et al. Cellular immune correlates of protection against symptomatic pandemic influenza. (2013). doi:10.1038/nm.3350

34. Wilkinson, T. M. et al. Preexisting influenza-specific CD4 + T cells correlate with disease protection against influenza challenge in humans. Nat. Med. 18, 274-280 (2012).

35. Provine, N. M. et al. MAIT cell activation augments adenovirus vector vaccine immunogenicity. Science (80-. ). 371, 521-526 (2021). 
Figure 1: Sequence variation in Spike glycoprotein

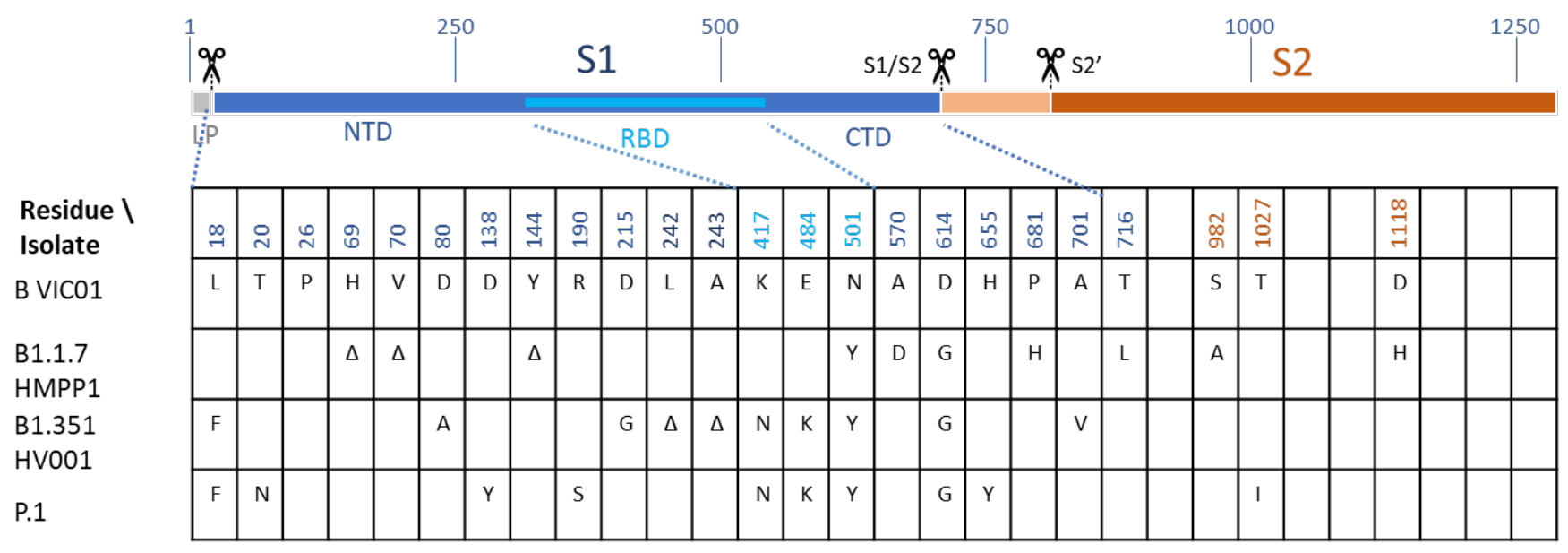


Figure 2a. Neutralization of sequence variants by convalescent sera

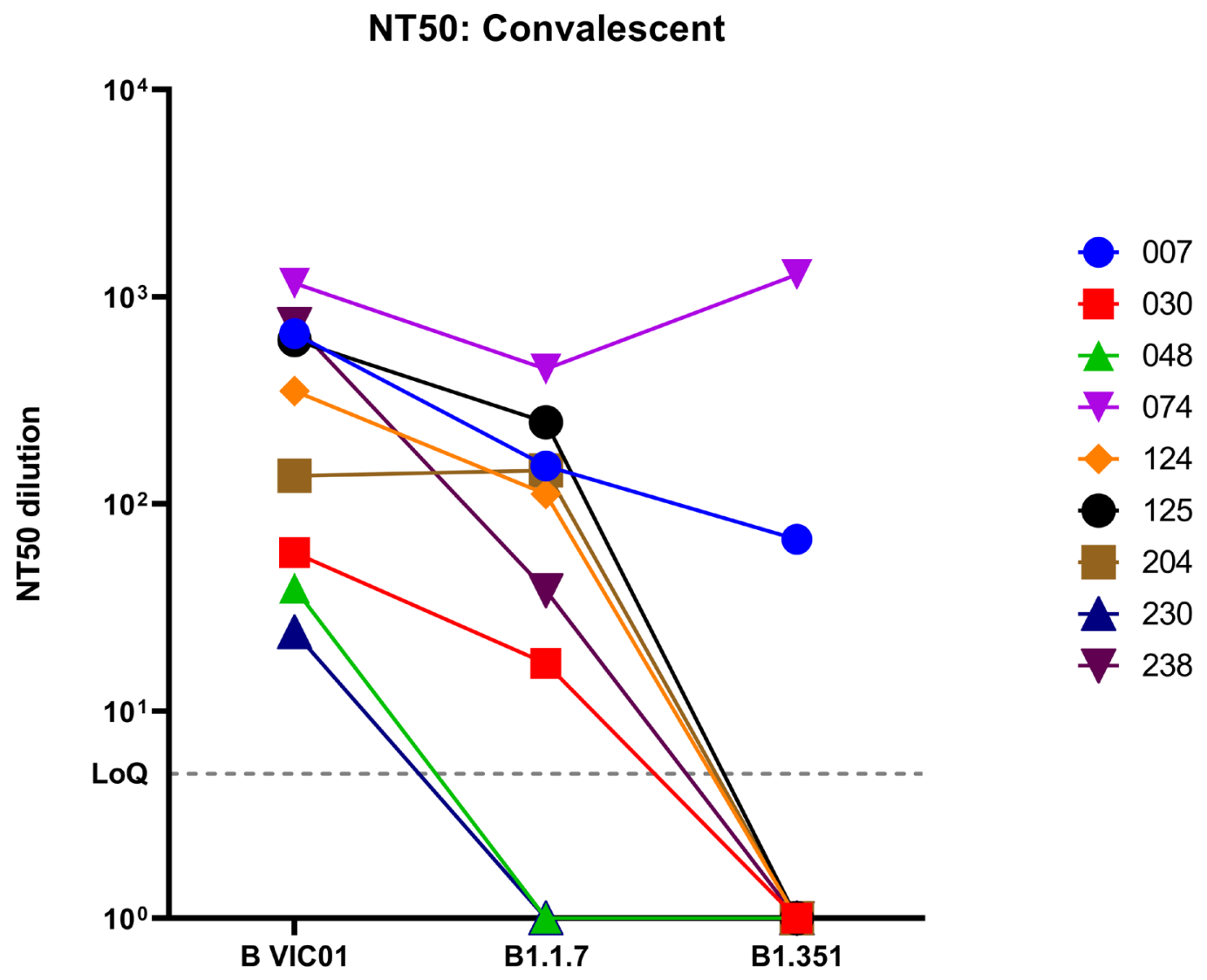


Figure $2 b$ \& $c$. Neutralization of sequence variants by post-vaccine ser
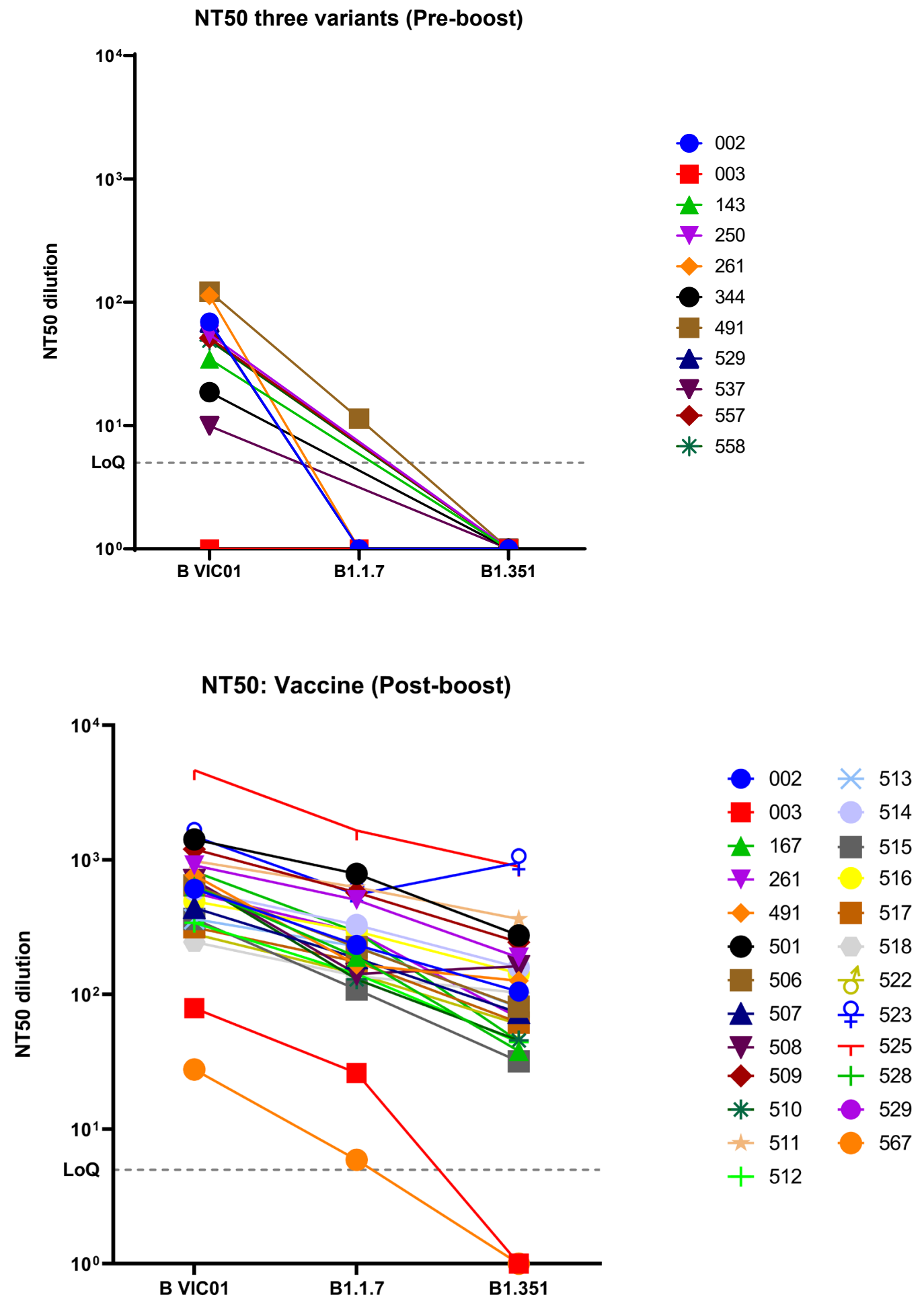
Figure $2 d \&$ e. Distribution of neutralization potencies

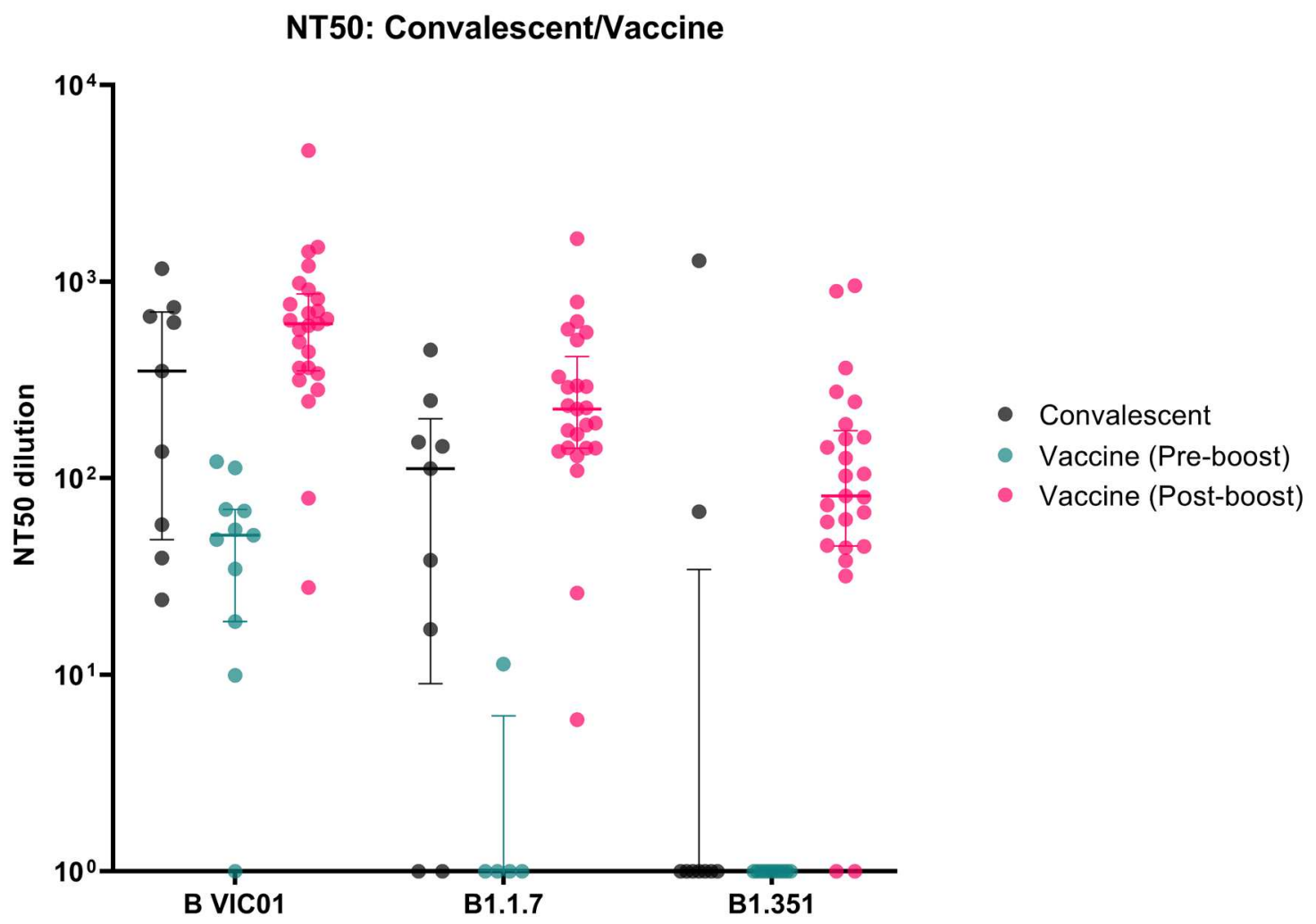

NT50: correlation

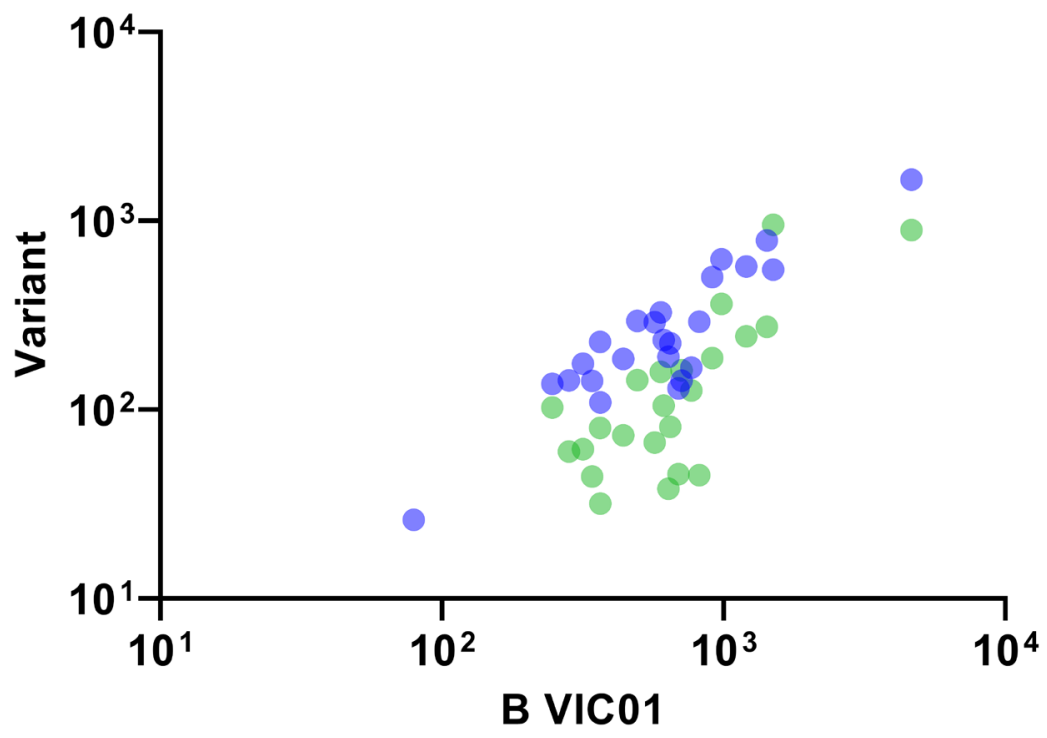

- B1.1.7

- B1.351 
Figure $2 f \& g$. Neutralization of VOCs by mAbs and reference antibody.

$\mathrm{F}$
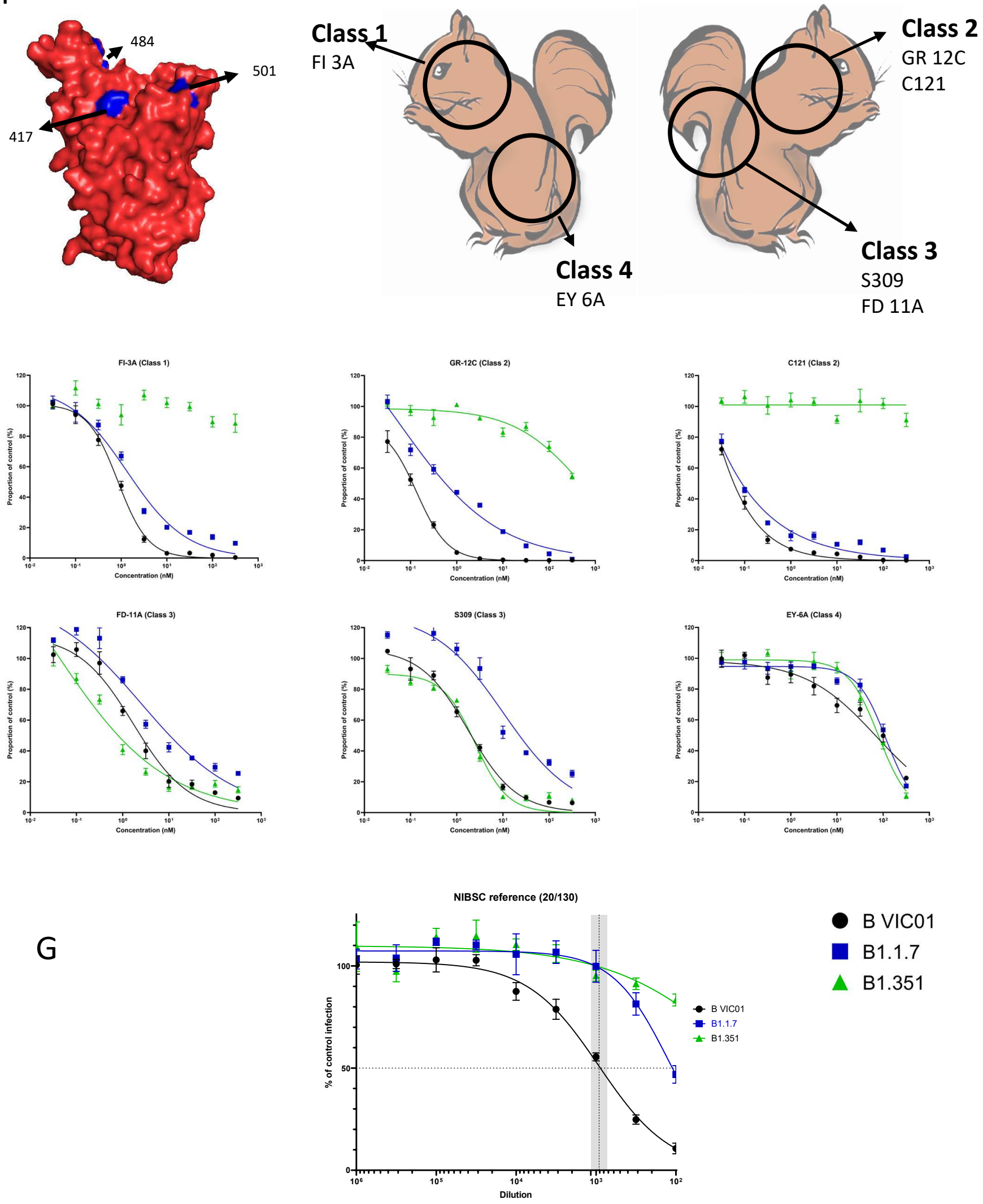
- B VIC01
- B1.1.7
$\triangle \mathrm{B} 1.351$

G 
Figure 3. Binding of serum antibody to coronavirus proteins (MSD assay)
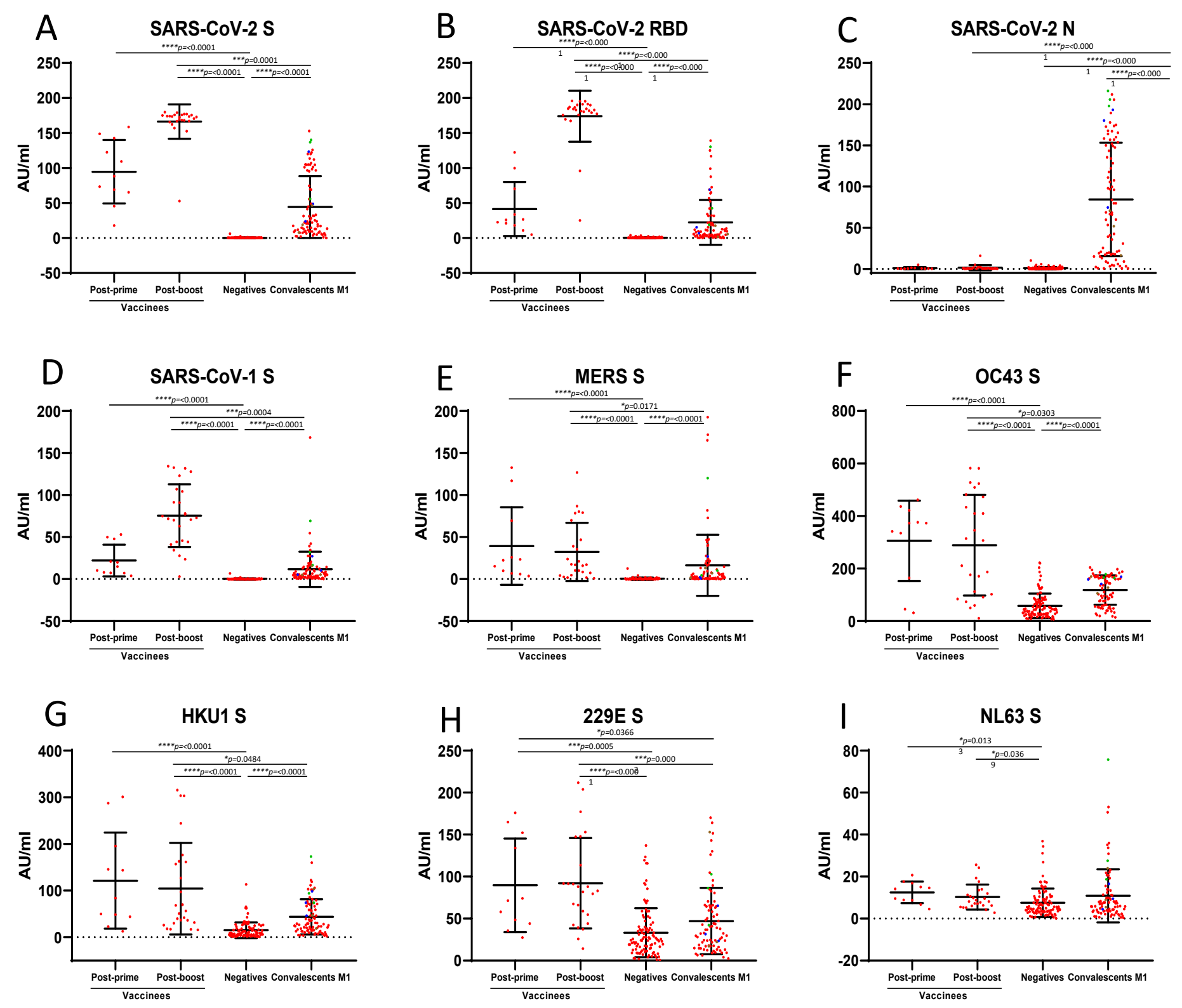
Figure 5: Cross-correlation of immune parameters

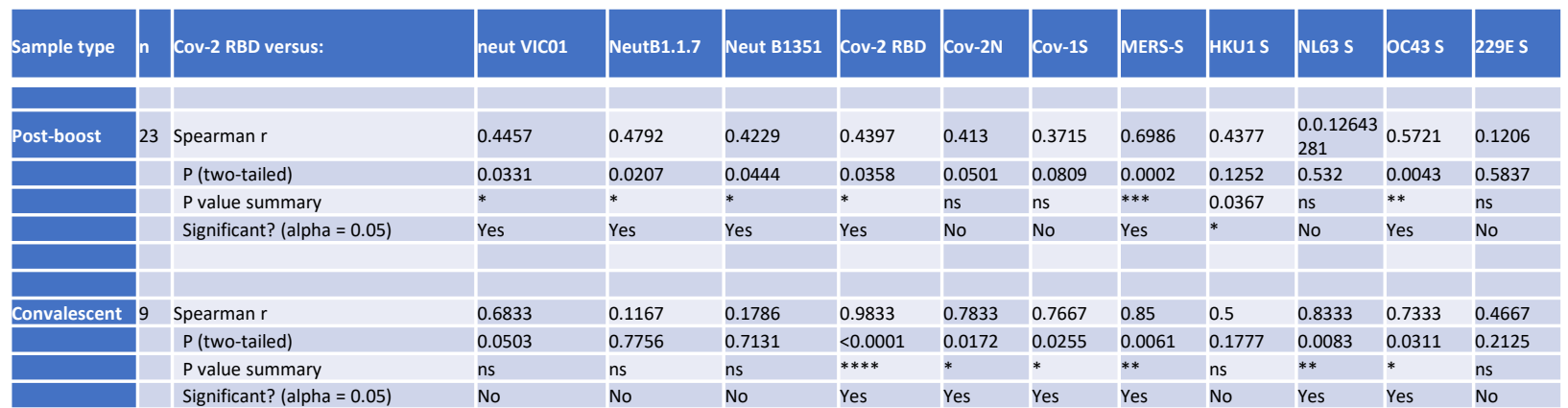

\section{post-boost}

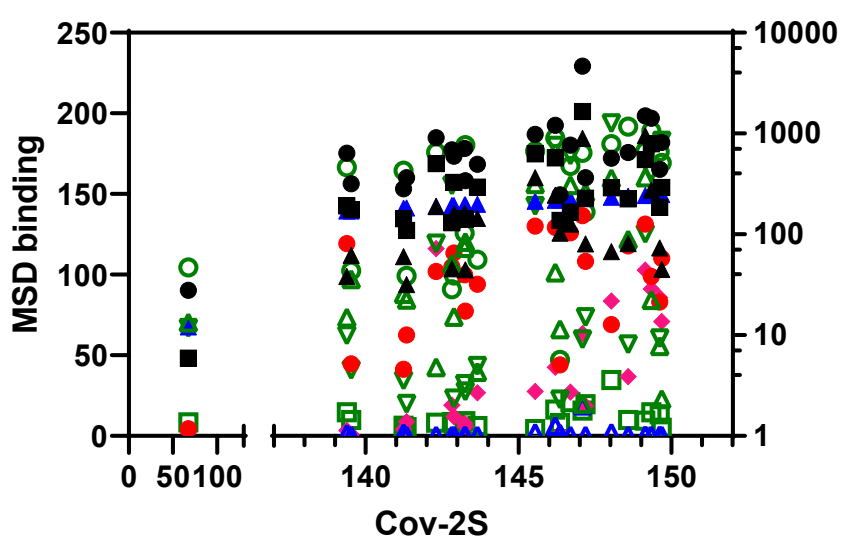
- Cov-2S
- OC43 S
- Neut VIC01
$\triangle$ Cov-2N
- NL63 S
- MERS-S
$\nabla$ HKU1S
- Neut B1.1.7
- Cov-1S
$\triangle$ 229E S

\section{convalescents}

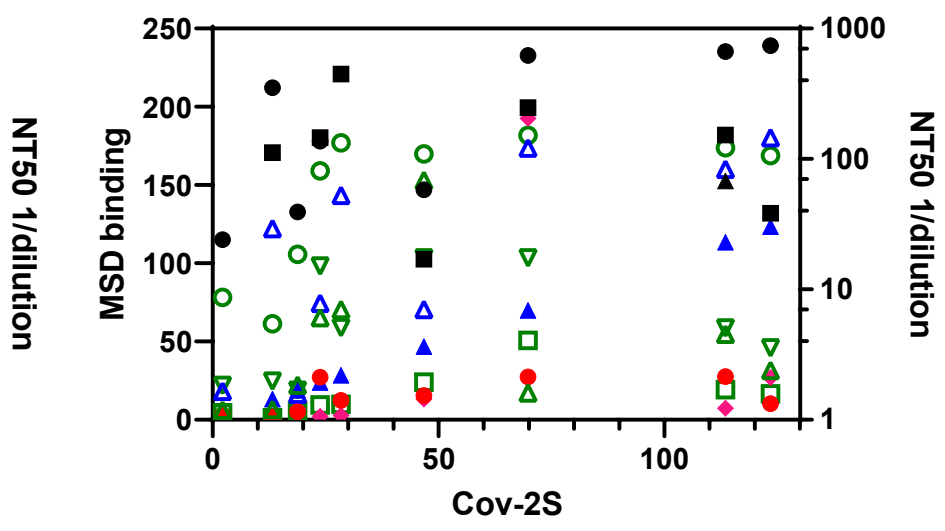


Figure S1. Individual neutralization curves
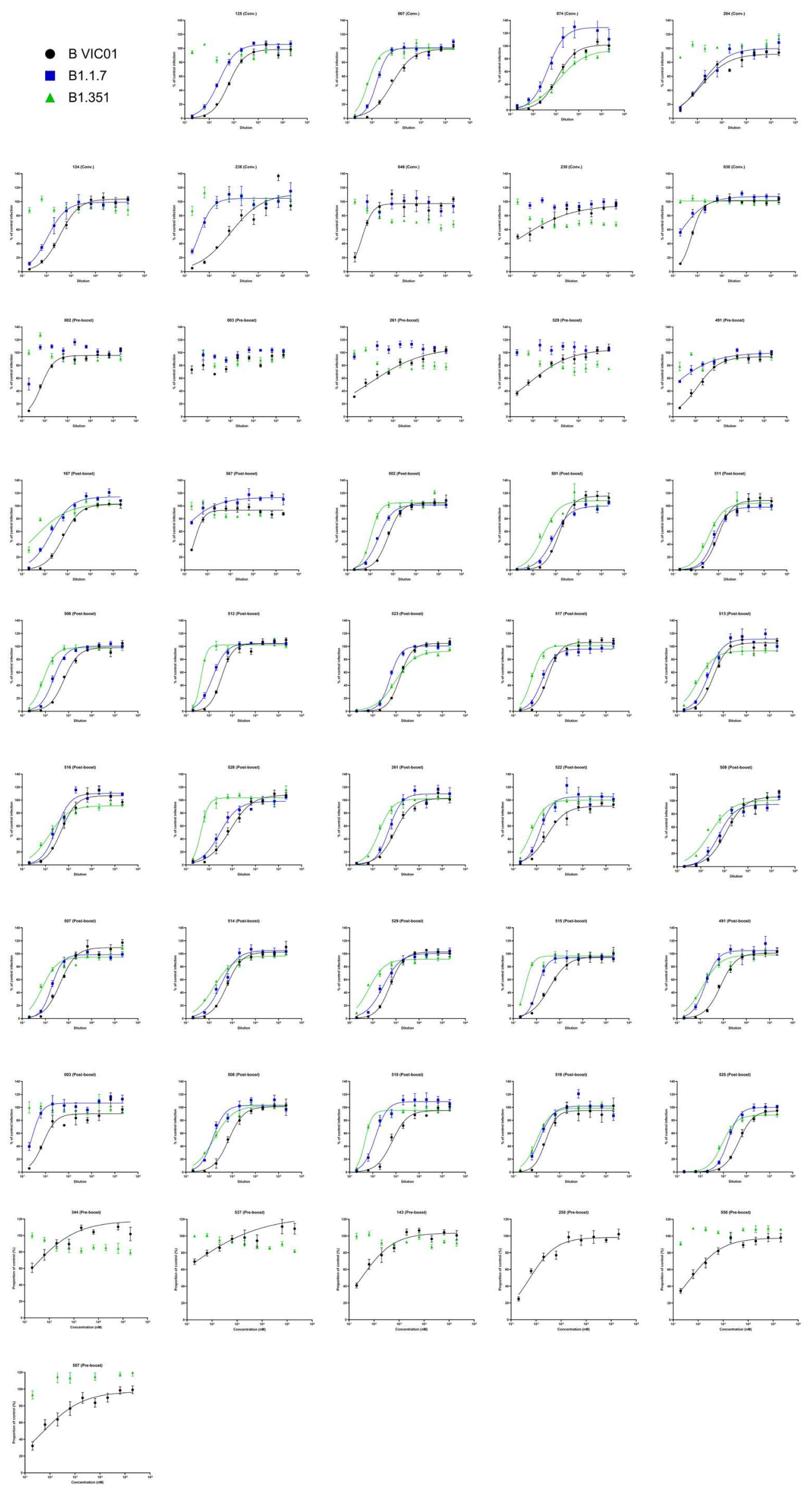


\section{Supplementary Table 1: Individual SARS-CoV-2 vaccinee T cell epitope data}

\begin{tabular}{|c|c|c|c|c|c|c|c|c|c|c|c|c|c|c|c|c|c|c|c|c|c|c|}
\hline IDs & L18F & T2ON & P26S & $69 / 70$ & D80A & D138Y & Y144 & R190s & D215G & LH242 & R246I & K417N & E484K & N501Y & A570D & H655Y & $\mathrm{P} 681 \mathrm{H}$ & A701V & 77161 & S982A & T1027| & $\mathrm{D} 1118 \mathrm{H}$ \\
\hline 002 & 0 & 0 & 0 & 5 & 80 & 70 & 15 & 0 & 0 & 0 & 10 & 0 & 30 & 15 & 20 & 0 & 0 & 0 & 25 & 0 & 0 & 0 \\
\hline 003 & 3 & 0 & 5 & 0 & 0 & 5 & 5 & 5 & 5 & 0 & 8 & 0 & 0 & 3 & 8 & 5 & 0 & 0 & 8 & 28 & 10 & 5 \\
\hline 167 & 0 & 0 & 0 & 0 & 0 & 0 & 0 & 0 & 0 & 0 & 0 & 0 & 0 & 0 & 0 & 0 & 0 & 0 & 5 & 0 & 0 & 0 \\
\hline 261 & 3 & 0 & 10 & 3 & 3 & 3 & 0 & 0 & 5 & 0 & 5 & 13 & 3 & 5 & 0 & 5 & 5 & 3 & 0 & 8 & 3 & 13 \\
\hline 491 & 0 & 0 & 0 & 0 & 0 & 0 & 25 & 0 & 0 & 0 & 0 & 5 & 0 & 0 & 0 & 0 & 0 & 0 & 0 & 0 & 0 & 0 \\
\hline 501 & 3 & 3 & 8 & 10 & 65 & 75 & 5 & 3 & 15 & 8 & 10 & 50 & 8 & 13 & 0 & 0 & 13 & 0 & 3 & 8 & 33 & 10 \\
\hline 506 & 5 & 0 & 0 & 10 & 0 & 10 & 0 & 0 & 25 & 10 & 25 & 0 & 5 & 0 & 0 & 15 & 0 & 5 & 15 & 5 & 0 & $\overline{0}$ \\
\hline 507 & 15 & 5 & 0 & 20 & 10 & 25 & 10 & 5 & 0 & 25 & 35 & 20 & 10 & 10 & 5 & 20 & 0 & 10 & 15 & 25 & 15 & 0 \\
\hline 508 & 15 & 5 & 5 & 15 & 20 & 10 & 20 & 20 & 10 & 0 & 10 & 15 & 15 & 0 & 10 & 20 & 0 & 10 & 5 & 0 & 10 & 0 \\
\hline 510 & 0 & 30 & 20 & 0 & 60 & 0 & 0 & 0 & 15 & 10 & 5 & 10 & 5 & 15 & 10 & 5 & 20 & 0 & 35 & 0 & 10 & 15 \\
\hline 511 & 0 & 3 & 23 & 8 & 18 & 23 & 18 & 38 & 20 & 15 & 28 & 18 & 10 & 20 & 23 & 15 & 50 & 5 & 25 & 15 & 18 & 23 \\
\hline 512 & 8 & 3 & 8 & 0 & 5 & 20 & 10 & 0 & 30 & 0 & 13 & 13 & 363 & 163 & 0 & 0 & 20 & 15 & 65 & 5 & 0 & 15 \\
\hline 513 & 0 & 0 & 0 & 5 & 3 & 0 & 0 & 20 & 0 & 10 & 0 & 18 & 0 & 0 & 0 & 0 & 3 & 0 & 3 & 0 & 5 & 5 \\
\hline 514 & 0 & 5 & 5 & 0 & 10 & 5 & 15 & 45 & 5 & 10 & 0 & 5 & 0 & 5 & 15 & 15 & 5 & 10 & 15 & 0 & 5 & 10 \\
\hline 515 & 0 & 0 & 0 & 18 & 5 & 3 & 0 & 5 & 0 & 0 & 5 & 10 & 0 & 5 & 0 & 0 & 8 & 0 & 13 & 3 & 10 & $\overline{3}$ \\
\hline 516 & 3 & 0 & 0 & 38 & 0 & 5 & 0 & 5 & 3 & 3 & 3 & 8 & 5 & 0 & 5 & 5 & 5 & 0 & 8 & 0 & 8 & $\underline{0}$ \\
\hline 517 & 3 & 5 & 18 & 3 & 5 & 5 & 3 & 0 & 10 & 10 & 0 & 3 & 3 & 0 & 5 & 0 & 8 & 3 & 8 & 0 & 8 & 3 \\
\hline 518 & 0 & 0 & 0 & 0 & 0 & 0 & 0 & 0 & 0 & 0 & 0 & 0 & 0 & 0 & 0 & 0 & 0 & 0 & 0 & 0 & 0 & $\overline{0}$ \\
\hline 518 & 3 & 3 & 0 & 3 & 0 & 0 & 0 & 0 & 5 & 3 & 0 & 10 & 0 & 0 & 3 & 8 & 3 & 0 & 0 & 3 & 0 & $\overline{0}$ \\
\hline 522 & 3 & 5 & 10 & 10 & 5 & 10 & 5 & 8 & 35 & 18 & 10 & 10 & 8 & 8 & 5 & 13 & 3 & 5 & 5 & 8 & 10 & 8 \\
\hline 525 & 0 & 0 & 0 & 0 & 0 & 0 & 3 & 8 & 0 & 0 & 5 & 0 & 0 & 10 & 0 & 0 & 0 & 0 & 0 & 0 & 5 & $\overline{0}$ \\
\hline 528 & 0 & 0 & 5 & 5 & 5 & 10 & 5 & 15 & 5 & 10 & 5 & 0 & 10 & 10 & 5 & 0 & 0 & 15 & 0 & 5 & 0 & 5 \\
\hline 529 & 5 & 5 & 5 & 3 & 38 & 15 & 23 & 5 & 3 & 0 & 10 & 5 & 10 & 13 & 8 & 3 & 3 & 0 & 5 & 0 & 5 & 13 \\
\hline 567 & 0 & 0 & 5 & 5 & 0 & 10 & 0 & 55 & 13 & 0 & 0 & 25 & 0 & 43 & 0 & 8 & 0 & 20 & 0 & 0 & 5 & 10 \\
\hline
\end{tabular}

S2: Ex vivo IFN- $\gamma$ ELISpot T cell responses to 22 mutation peptide sites relative to prototype strain are shown. Each row represents a single individual, and each column represents peptides in prototype strain that corresponds with mutations in B1.1.7, B1.351 and P.1. T cells that target epitopes located at the sites in B1.1.7, B1.351 and P.1 are shown in blue. N=24, results reported as SFC $/ 10^{6}$ PBMC. 
Figures

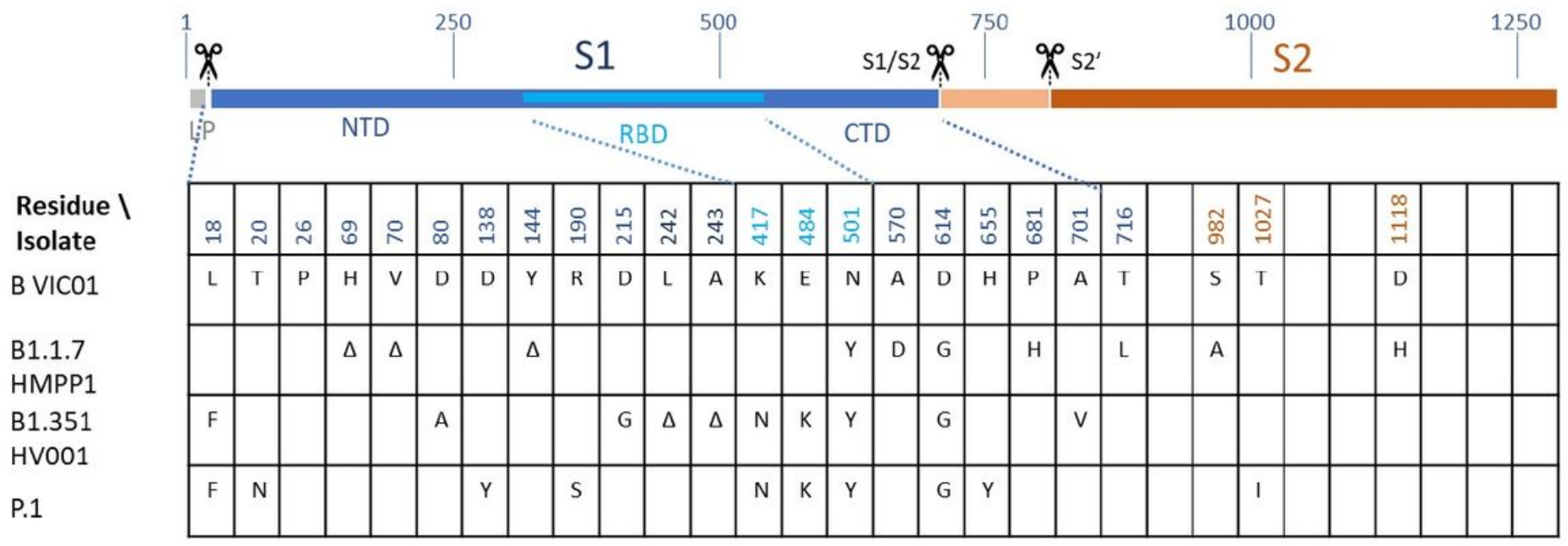

Figure 1

Sequence variation in Spike glycoprotein
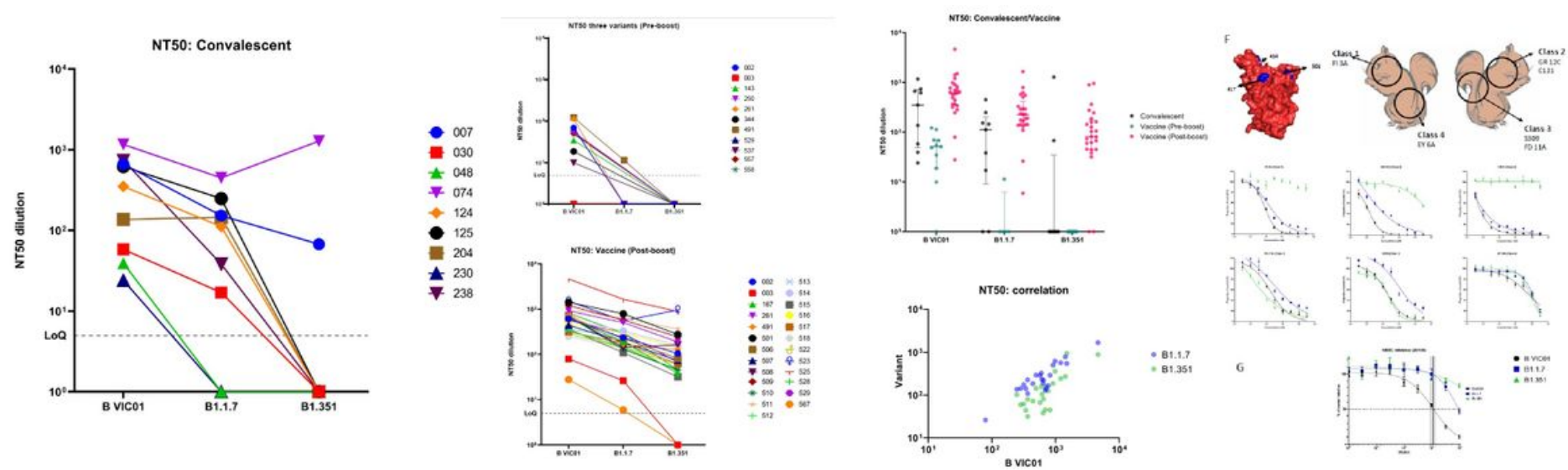

\section{Figure 2}

a. Neutralization of sequence variants by convalescent sera. b \& c. Neutralization of sequence variants by post-vaccine sera. $d \&$ e. Distribution of neutralization potencies. $f \& g$. Neutralization of VOCs by mAbs and reference antibody. 

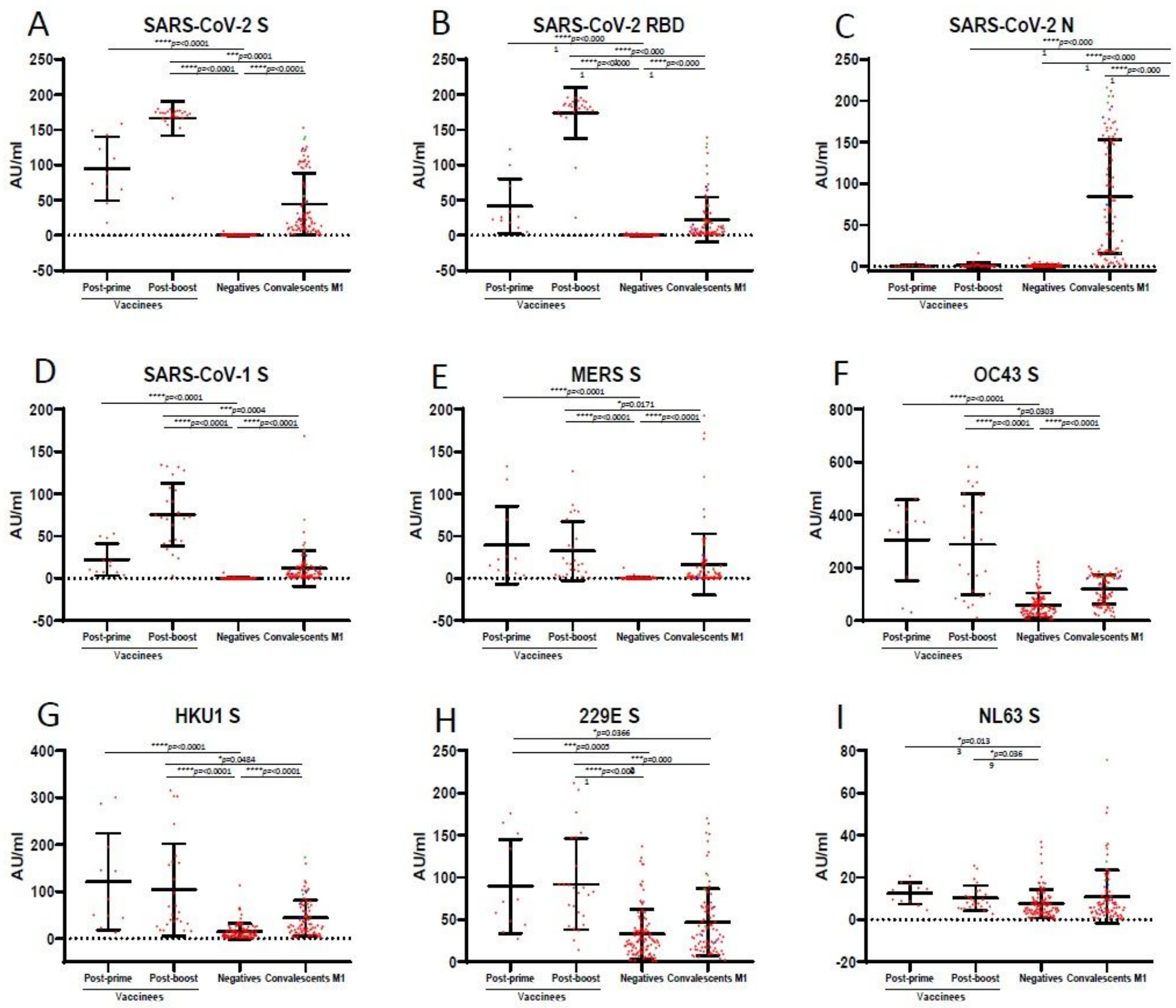

Figure 3

Binding of serum antibody to coronavirus proteins (MSD assay) 


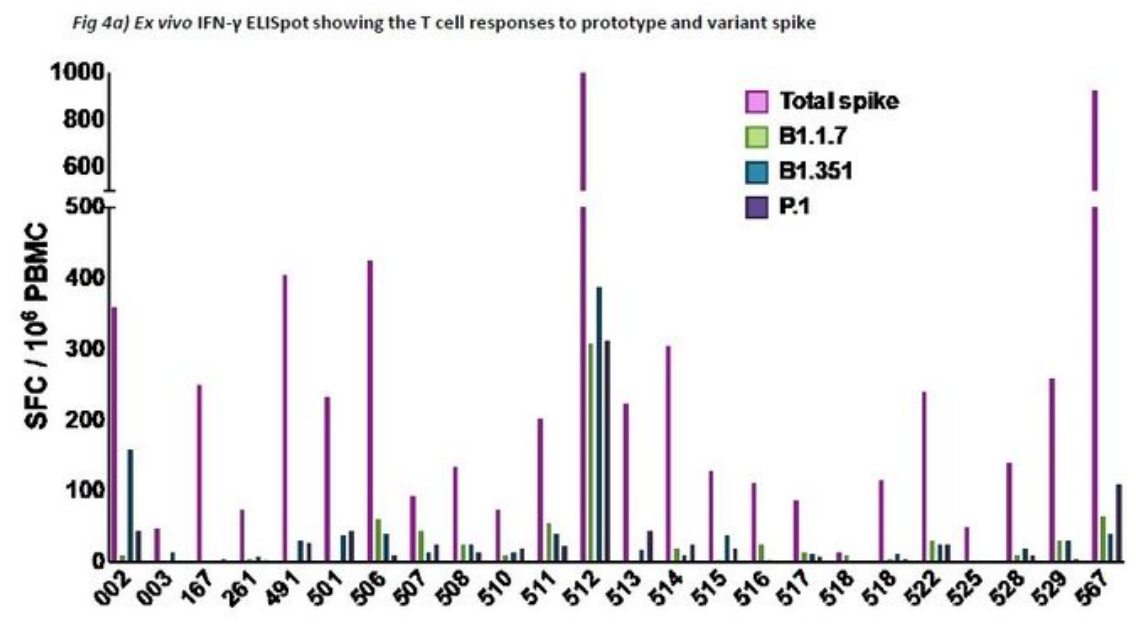

Fig 4b) Percentage contribution of SARS-CoV-2 variants to the total spike response in SARS-CoV-2 vaccinated healthy volunteers
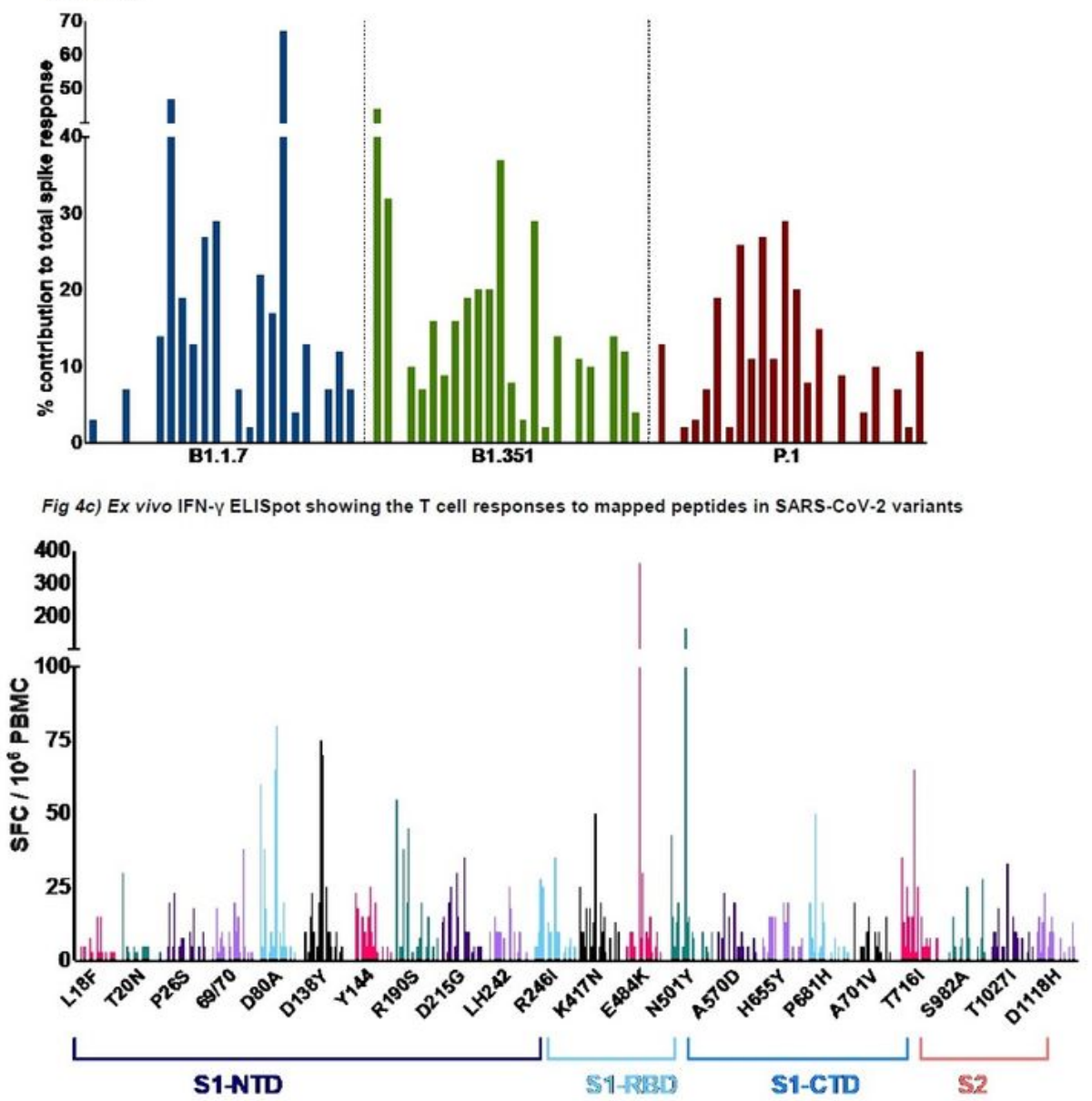

Figure 4

ELISPOT responses to prototype, B1.1.7, B1.351 and P1. 


\begin{tabular}{|c|c|c|c|c|c|c|c|c|c|c|c|c|c|}
\hline Sample type & in & Cov-2 RBD versus: & neut VICO1 & NeutB1.1.7 & Neut B1351 & Cov-2 RBD & COV-2N & Cov-1S & MERS-S & HKU1S & NL63 S & oc43s & 229ES \\
\hline \multirow[t]{4}{*}{ Post-boost } & 23 & Spearman $r$ & 0.4457 & 0.4792 & 0.4229 & 0.4397 & 0.413 & 0.3715 & 0.6986 & 0.4377 & $\begin{array}{l}0.0 .12643 \\
281\end{array}$ & 0.5721 & 0.1206 \\
\hline & & $P$ (two-tailed) & 0.0331 & 0.0207 & 0.0444 & 0.0358 & 0.0501 & 0.0809 & 0.0002 & 0.1252 & 0.532 & 0.0043 & 0.5837 \\
\hline & & $P$ value summary & * & * & $*$ & * & ns & ns & $* * *$ & 0.0367 & ns & $* *$ & ns \\
\hline & & Significant? (alpha $=0.05$ ) & Yes & Yes & Yes & Yes & No & No & Yes & * & No & Yes & No \\
\hline & & & & & & & & & & & & & \\
\hline \multirow[t]{4}{*}{ Convalescent } & 9 & Spearman $\mathrm{r}$ & 0.6833 & 0.1167 & 0.1786 & 0.9833 & 0.7833 & 0.7667 & 0.85 & 0.5 & 0.8333 & 0.7333 & 0.4667 \\
\hline & & P (two-tailed) & 0.0503 & 0.7756 & 0.7131 & $<0.0001$ & 0.0172 & 0.0255 & 0.0061 & 0.1777 & 0.0083 & 0.0311 & 0.2125 \\
\hline & & P value summary & ns & ns & ns & $* * * *$ & $*$ & * & $* *$ & ns & $* *$ & * & ns \\
\hline & & Significant? (alpha $=0.05$ ) & No & No & No & Yes & Yes & Yes & Yes & No & Yes & Yes & No \\
\hline
\end{tabular}

\section{post-boost}
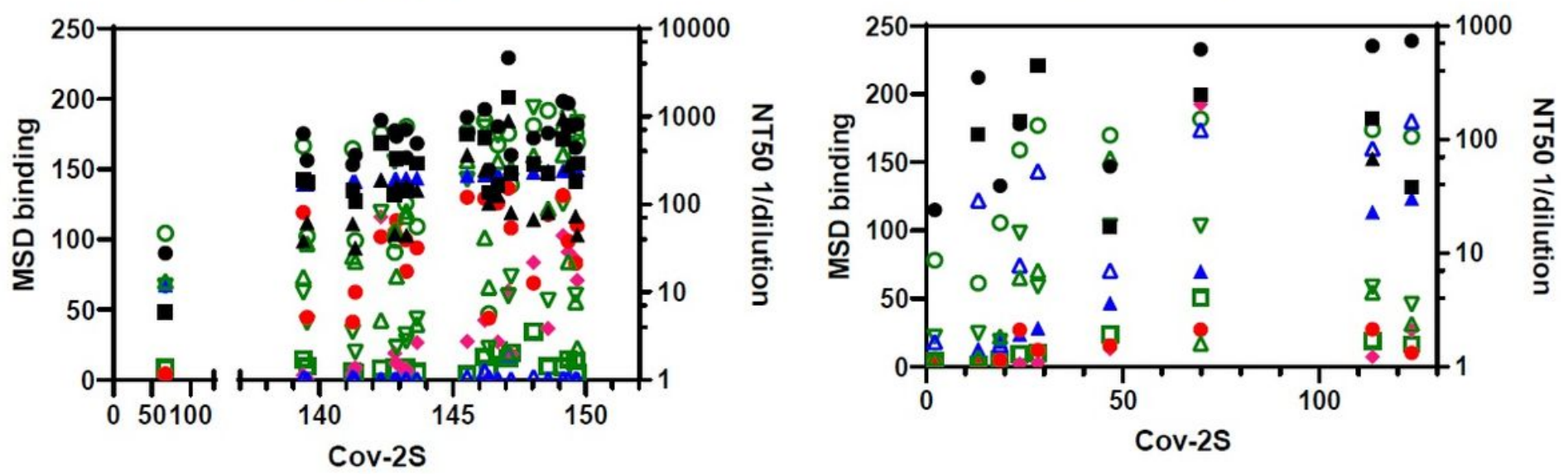
- Cov-2S
- Oc43s
- Neut VIC01
$\Delta$ Cov-2N
- NL63S
- MERS-S
$\nabla$ HKU1S
- Cov-1S
$\triangle$ 229E S
- Neut B1.1.7
- Neut B1.351

Figure 5

Cross-correlation of immune parameters 